\title{
Synthese von hierarchischen Nanostrukturen durch elastokapillare Selbstorganisation
}

Michaël De Volder* und A. John Hart

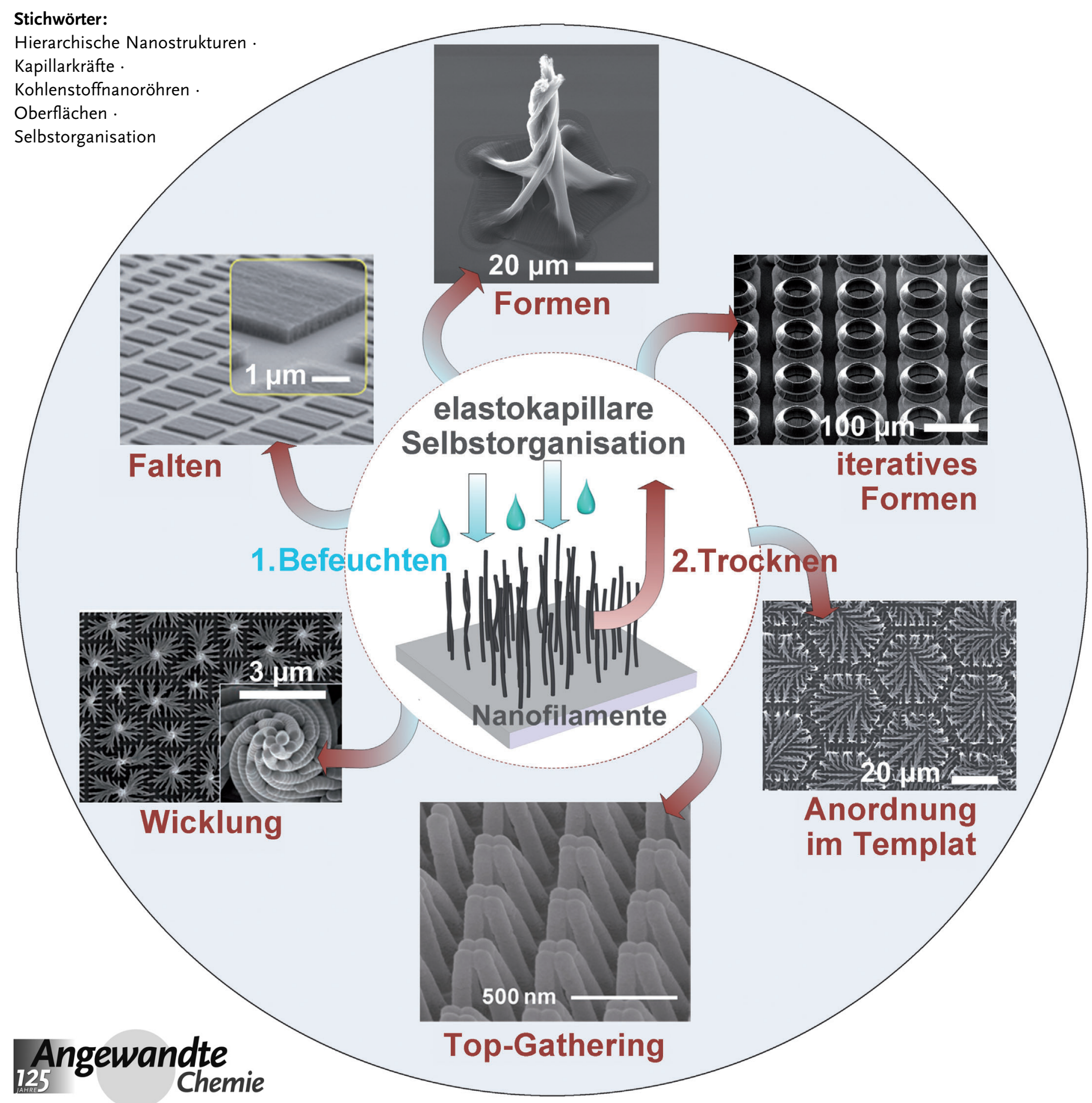


O berflächen, die mit nanoskaligen Filamenten wie Siliciumnanodrähten und Kohlenstoffnanoröhren beschichtet sind, sind potenziell hochinteressant für Hochleistungsbatterien und Kondensatorelektroden, Photovoltaik, elektrische Schaltungen, Substrate für Zellzüchtungen, Trockenklebstoffe und andere intelligente Materialien. Viele diese Anwendungen erfordern eine nasse Umgebung oder umfassen Verarbeitungsschritte in Lösung. Die durch diese nassen Umgebungen erzeugten Kapillarkräfte können einerseits zu einer unerwünschten Aggregation der nanoskaligen Filamente führen, andererseits kann über die Steuerung der Kapillarkräfte jedoch ein Manipulieren dieser Filamente zur Bildung diskreter Aggregate und neuer hierarchischer Strukturen möglich werden. Viele neuere Arbeiten deuten darauf hin, dass die elastokapillare Selbstorganisation von Nanofilamenten eine vielseitige und skalierbare Methode zum Aufbau komplexer und robuster Oberflächenarchitekturen sein kann. Mit diesem Aufsatz möchten wir zum Verständnis der elastokapillaren Selbstorganisation als Herstellungsmethode beitragen und deren Anwendungspotenzial vorstellen. Wir erläutern die Grundlagen und klassifizieren typische Anwendungen und Oberflächendesigns für Nanodrähte, Nanoröhren und Nanosäulen aus verschiedensten Materialien. Schließlich diskutieren wir beispielhafte Anwendungen und zukünftige Möglichkeiten, um neue Oberflächen über die elastokapillare Selbstorganisation von Nanofilamenten zu realisieren.

\section{Aus dem Inhalt}

\begin{tabular}{l} 
1. Einleitung \\
$\begin{array}{l}\text { 2. Der Mechanismus der } \\
\text { elastokapillaren Selbst- } \\
\text { organisation }\end{array}$ \\
$\begin{array}{l}\text { 3. Klassifizierung von } \\
\text { elastokapillaren } \\
\text { Selbstorganisationsmethoden }\end{array}$ \\
$\begin{array}{l}\text { 4. Klassifizierung von } \\
\text { Oberflächenmustern nach } \\
\text { elastokapillarer } \\
\text { Selbstorganisation }\end{array}$ \\
\hline \begin{tabular}{l} 
5. Prozesssteuerung \\
\hline 6. Anwendungen
\end{tabular} \\
\hline
\end{tabular}

7. Zusammenfassung und Ausblick 2482

\section{Einleitung}

Oberflächen, die mit nanoskaligen Filamenten mit hohem Aspektverhältnis beschichtet sind, z. B. mit Polymernanopfeilern, Kohlenstoffnanoröhren (CNT) und Halbleiter-Nanodrähten, bieten einzigartige Eigenschaften, z. B. eine abstimmbare mechanische Federung und eine große Oberfläche für den Ladungstransport oder für elektrochemische Wechselwirkungen. ${ }^{[1]}$ Infolgedessen sind faserige Oberflächen für bestimmte Anwendungen äußerst attraktiv, z.B. für Superkondensatoren, ${ }^{[2,3]}$ biomimetische Klebstoffe, ${ }^{[4,5]}$ Solarzellen, ${ }^{[6,7]}$ superhydrophobe Oberflächen, ${ }^{[8]}$ Wärmetauscher, ${ }^{[9]}$ DNA-Separation, ${ }^{[10]}$ biomedizinische Diagnostika, ${ }^{[11]}$ Materialien für Schnittstellen mit biologischen Zellen ${ }^{[12,13]}$ und anisotrope Oberflächen. ${ }^{[14]}$

Dabei muss unbedingt berücksichtigt werden, dass viele dieser Bauelemente während der Bildung der Fasern, bei anschließenden Herstellungsschritten ${ }^{[15]}$ oder bei der endgültigen Anwendung mit einer nassen Umgebung in Kontakt kommen. ${ }^{[12]}$ Daher bedarf es Überlegungen, wie Kapillarkräfte die Morphologie der Filamente auf der Oberfläche beeinflussen können. Aufgrund der mechanischen Verformbarkeit von Nanofilamenten mit hohem Aspektverhältnis und dem Zusammenhang zwischen Kapillardruck und Meniskusrundung kann ein Kontakt mit Flüssigkeiten leicht diese Strukturen beeinflussen. Darüber hinaus kann die relative Stärke von elektrostatischen und adhäsiven Wechselwirkungen zwischen den Nanostrukturen (z.B. Van-der-
Waals-Kräften) deren Anordnung verändern, wenn die Flüssigkeit verdampft. ${ }^{[16]}$

Während die kapillare Aggregation anfänglich als nachteilig für die Verarbeitung von lithographisch erzeugten, hoch aufgelösten Polymerstrukturen beschrieben wurde, ${ }^{[15]}$ zeigen nun Fortschritte bei der kontrollierten Gestaltung von Nanostrukturen, dass sich Kapillarwechselwirkungen in einem vielseitigen und skalierbaren Herstellungsschritt nutzen lassen. Zum Beispiel wurde das Befeuchten und Trocknen von vertikal angeordneten Kohlenstoffnanoröhren (CNT) genutzt, um deren Packungsdichte zu erhöhen, ${ }^{[17-22]}$ periodische Überstrukturen zu bilden, ${ }^{[23-25]}$ Dünnschichten zu horizontalen Lagen und multidirektionalen Schaltkreisen zu reorientieren $^{[26-30]}$ und 3D-Mikroarchitekturen herzustellen. ${ }^{[28,31-35]}$ Ähnliche Prinzipien wurden verwendet, um Nanofilamente $\mathrm{zu}$ manipulieren, die aus $\mathrm{Si},{ }^{[36-39]} \mathrm{Au},{ }^{[40]} \mathrm{ZnO},{ }^{[41]}$ $\mathrm{Cu},{ }^{[38]}$ Hydrogel, ${ }^{[1,42]}$ Epoxidharz ${ }^{[1,16]}$ PMMA,${ }^{[12,43,44]}$ PET, ${ }^{[45]}$ cyclischen Olefin-Copolymeren, ${ }^{[12]}$ Polyurethan, ${ }^{[1]}$ PDMS, ${ }^{[46]}$

[**] Dr. M. De Volder

imec und Department of Mechanical Engineering, KULeuven Heverlee (Belgien)

E-Mail: michael.devolder@imec.be

Dr. M. De Volder

School of Engineering and Applied Sciences, Harvard University Cambridge, MA (USA)

Prof. A. J. Hart

Department of Mechanical Engineering, University of Michigan Ann Arbor, MI (USA) 
verschiedenen Photolacken ${ }^{[15,47,48]}$ und sogar biologischen Fasern bestehen. ${ }^{[49]}$

Dieser Aufsatz beschäftigt sich mit Methoden, Mechanismen und Anwendungen der elastokapillaren Selbstorganisation von vertikalen Nanofilamenten auf Substraten. Mit dieser Arbeit möchten wir: 1) die Methoden erläutern und klassifizieren, die derzeit bei der elastokapillaren Selbstorganisation von Nanofilamenten angewendet werden; 2) eine umfassende Übersicht darüber bieten, wie diese Methoden zur Schaffung von ungeordneten und geordneten 2D- und 3D-Strukturen eingesetzt werden können; 3) physikalische Modelle diskutieren sowie praktische Orientierungshilfen zur Entwicklung und Optimierung des elastokapillaren Selbstorganisationsprozesses vorstellen; 4) aktuelle und zukünftige Anwendungen in der Nano-/Mikroherstellung und der Oberflächentechnik identifizieren. Neben dem aktuellen Stand der Technik umfasst dieser Aufsatz auch unveröffentlichte Beobachtungen aus unserer eigenen Forschung zur kapillaren Selbstorganisation. Darüber hinaus existieren auch Berichte, nach denen die kapillare Selbstorganisation zur Manipulation diskreter Partikel und strukturierter Dünnschichten (einschließlich „Mikro-Origami“-Strukturen) verwendet wurde. Allerdings geht dieses Thema über den Umfang dieses Aufsatzes hinaus, und wir verweisen daher auf weitere Übersichten. ${ }^{[50-54]}$

\section{Der Mechanismus der elastokapillaren Selbst- organisation}

Wir definieren elastokapillare Selbstorganisation (die gelegentlich auch als elastokapillare Aggregation bezeichnet wird) als mechanische Verformung und Veränderung der Anordnung von Filamenten durch den Einfluss von Kapillarkräften beim Benetzen mit einer Flüssigkeit oder beim Verdampfen derselben. Bei zurückweichendem Meniskus wird auf benachbarte Filamente eine Querkraft ausgeübt. Sind diese Kapillarkräfte stärker als die elastische Rückstellkraft der deformierten Filamente, aggregieren diese und gelangen miteinander in Kontakt. Übertreffen die Oberflächenkräfte zwischen den in Kontakt stehenden Filamenten dann die elastischen Rückstellkräfte, kann die aggregierte Konfiguration nach dem Verdampfen der Flüssigkeit stabil sein. Ein grundlegendes Beispiel findet sich in Abbildung $1 \mathrm{a}$, bei dem eine quadratische Gitteranordnung von EpoxidharzMikrosäulen zu Clustern von vier an der Spitze aufeinan-

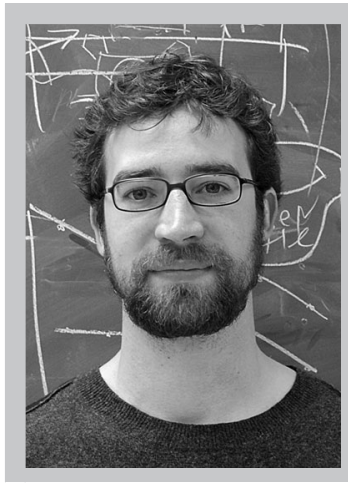

Michaël De Volder promovierte an der KULeuven, Belgien, sowie am Tokyo Institute of Technology, Japan, mit Arbeiten im Bereich der Mikrosystemtechnologie. Anschließend absolvierte er Postdoktorate im Bereich der Kohlenstoffnanoröhren und kapillaren Selbstorganisation an der University of Michigan, am MIT und an der Harvard University sowie am imec und der KULeuven. Er ist Preisträger der Royal Academy of Science and Fine Arts, Belgien und erhielt mehrere renommierte Auszeichnungen aus Wissenschaft und Industrie. dertreffenden Säulen aggregiert. In diesem Fall sind die Oberflächenkräfte zwischen den in Kontakt stehenden Säulen (an den Spitzen) stärker als die durch die Auslenkung der Säulen bedingten elastischen Kräfte, und die Cluster sind nach dem Rückzug der Flüssigkeit stabil.

Verschiedene analytische Modelle erlauben eine Vorhersage des aggregierten Zustands nach dem Trocknen. Zum Beispiel beobachteten Chandra et al., ${ }^{[1]}$ dass sich Säulen zusammenfinden, sobald ihre Spitzen die Flüssigkeitsoberfläche durchstoßen. Durch Gleichsetzen der elastischen und Kapillarkräfte leiten sie den minimal erforderlichen Elastizitätsmodul $E_{\text {crit }}$ ab, um einer elastokapillaren Aggregation zu widerstehen, ${ }^{[1]}$ d.h. bei einer gegebenen Oberflächenspannung zum Zusammenhalten der Säulen. Unter Verwendung von mit einem regelmäßigen Abstand angeordneten Nanofilamenten als Modellsystem (Abbildung 1a,b) ergibt sich Gleichung (1): $:^{[1]}$

$E_{\text {crit }}=\frac{32 \sqrt{2} \gamma \cos ^{2} \theta h^{3}}{3 d^{4}} f(r)$

Dabei ist $\theta$ der Kontaktwinkel, $\gamma$ die Oberflächenspannung, $h$ die Säulenhöhe, $d$ ist der Säulendurchmesser und $f(r)$ ist eine Funktion des Durchmessers und des Abstands der in Abbildung $1 \mathrm{~b}$ dargestellten Säulen.

Dieser Ansatz wurde erweitert, um das Clusterbildungsverhalten von Anordnungen mit einer großen Anzahl von Nanofilamenten zu verstehen. ${ }^{[1]}$ Wenn sich die Filamente einander entgegenneigen, nimmt der Abstand zwischen den Spitzen ab, was zu einem Abnehmen der Kapillarenergie und einer Zunahme der elastischen Biegeenergie führt. Sammelt sich eine Gruppe (ein Cluster) von Filamenten an einem gemeinsamen Punkt, müssen sich die äußeren Filamente stärker biegen als die inneren. Daher nimmt die Biegeenergie pro Filament mit zunehmender Clustergröße zu. Diesem kann nur durch die Oberflächenspannung von flexiblen (z.B. langen) Filamenten Rechnung getragen werden. Die resultierende Clustergröße, $N_{c}$, ist dann: $:^{[1]}$

$N_{c}=\frac{273 \cos ^{2} \theta \gamma h^{3}}{E d^{2} w^{2}}$

Dabei ist $\theta$ der Kontaktwinkel, $\gamma$ die Oberflächenspannung, $h$ die Säulenhöhe, $E$ der Elastizitätsmodul, $d$ der Säulendurchmesser und $w$ der Abstand zwischen benachbarten Säulen (unter Annahme eines quadratischen Gitters wie in Abbildung $1 \mathrm{~b}$ ).

Nachdem die Flüssigkeit verdampft und die Nanofilamente trocken sind, wird die Stabilität des aggregierten $\mathrm{Zu}$ stands durch die Adhäsion (z. B. Van-der-Waals-Wechselwirkungen) bestimmt. Denn im trockenen Zustand muss die elastische Energie durch die Filament-Filament-Wechselwirkungen ausgeglichen werden, und daher spielen diese eine Schlüsselrolle für die endgültige Geometrie der Aggregate. ${ }^{[16,46,56,57]}$ Mit anderen Worten bestimmt das Gleichgewicht zwischen Kapillaranziehung und elastischer Rückstellung die maximale Anzahl von Säulen, die zu einem Bündel aggregieren können, während das Gleichgewicht von Adhäsion und Elastizität bestimmt, wie viele Nanofilamente in der 
a)
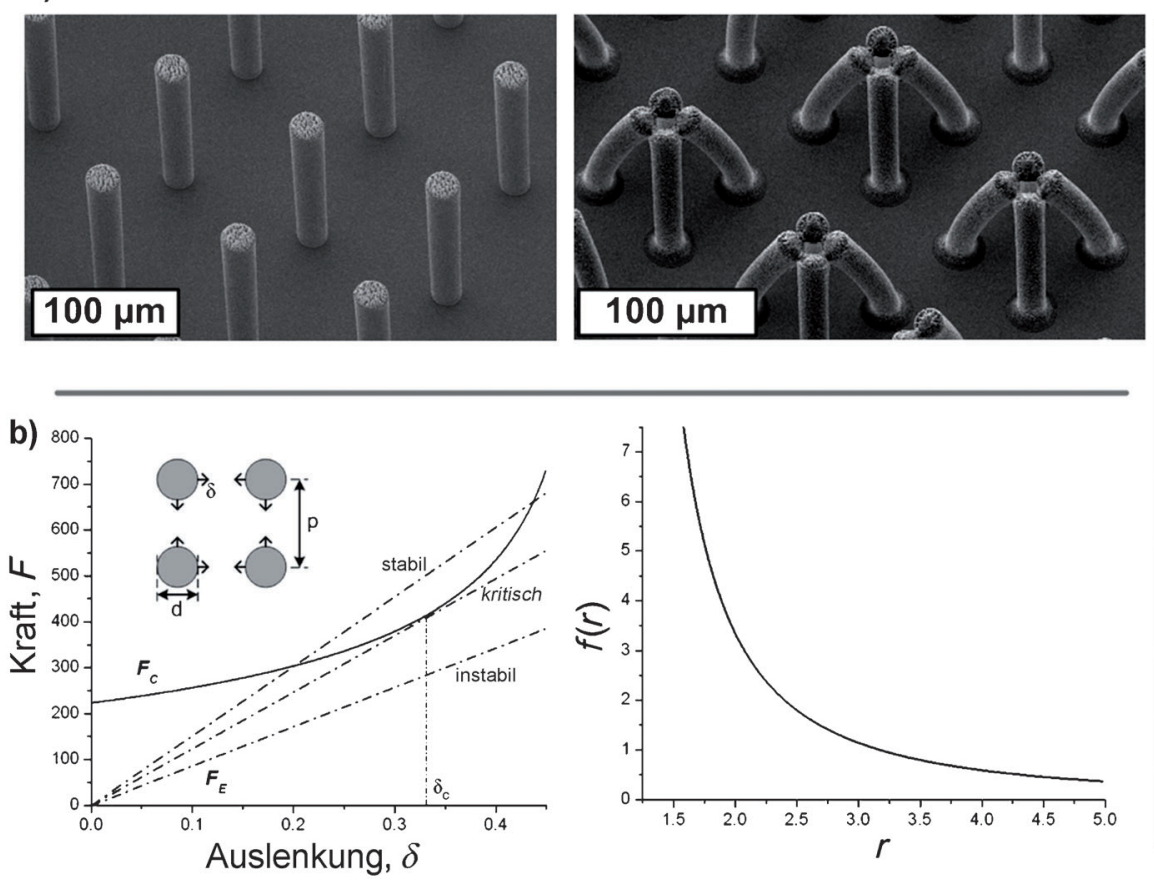

c)

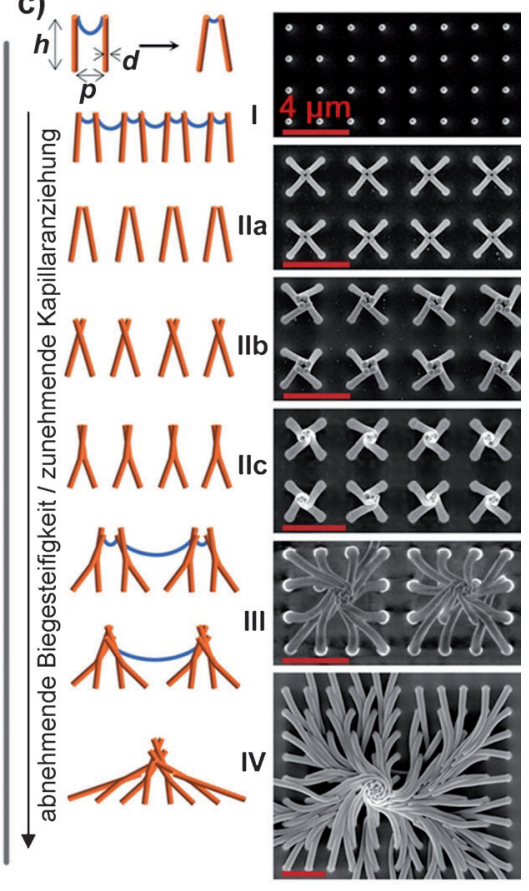

Abbildung 1. a) Epoxidharz-Mikrosäulen vor (links) und nach Aggregation (rechts) zu Gruppen von vier Säulen aufgrund kapillarer Selbstorganisation. b) Modell der Säulenaggregation zu Gruppen von vier Säulen [GI. (1)]. Das linke Diagramm zeigt die Kapillarwechselwirkungskraft $F_{C}$ und die elastische Rückstellkraft $F_{\mathrm{E}}$. Das rechte Diagramm zeigt $f$ als eine Funktion von $r=P / d$. Nachdruck mit Genehmigung aus Lit. [1], Copyright 2010 American Chemical Society. c) Entwicklung der Nanofilament-Cluster von Vierergruppen zu komplexen helikalen Anordnungen mit zunehmender Länge der Filamente (Maßstab $4 \mu \mathrm{m}$ ). Nachdruck mit Genehmigung aus Lit. [55], Copyright AAAS.

endgültigen Struktur nach dem Trocknen erhalten bleiben. ${ }^{[16,56]}$ Im weiteren Verlauf diskutieren wir, wie Filamente zu komplexen helikalen Borsten und anderen 3D-Geometrien aggregieren können, die in Abbildung $1 \mathrm{c}$ abgebildet sind. ${ }^{43,55,56]}$

Eine wesentliche Annahme dieser Modelle ist, dass die Filamente auf dem Substrat isoliert, parallel und starr fixiert vorliegen. Diese Annahmen treffen auf eine ganze Reihe technischer Nanofilamente $\mathrm{zu}$, da die meisten Herstellungsmethoden zu Anordnungen von vergleichsweise geraden Säulen mit gleichmäßigen Abständen führen. Solche Methoden umfassen das direkte Schreiben von Polymernanodrähten mit einem Elektronenstrahl in einem Photolack, ${ }^{[43]}$ das Replikatformen von Siliciumvorlagen zur Erzeugung von Polymersäulenanordnungen, ${ }^{[58]}$ anisotropes Ätzen von Siliciumstempeln ${ }^{[59,60]}$ und Züchtung von Nanodrähten und Nanoröhren durch chemische Gasphasenabscheidung (CVD). ${ }^{[61-63]}$ Die hohe Flexibilität der Filamente mit sehr kleinem Durchmesser wie Kohlenstoffnanoröhren (CNT) führt jedoch $\mathrm{zu}$ einer welligen und verfilzten Geometrie, wodurch das Aggregationsverhalten auch von der Organisation und der Gesamtform der Filamentpopulation abhängig wird. Im Folgenden werden wir uns auch auf solche großen Populationen aus verfilzten Nanofilament-,,Wäldern“ beziehen. Weitere Einschränkungen werden später beschrieben; an dieser Stelle lautet unsere Botschaft jedoch, dass die kapillare Selbstorganisation durch die Flüssigkeit $(\gamma, \theta)$, das Säulenmaterial $(E$, Oberflächenchemie) und die Geometrie $(d, h, w)$ beeinflusst wird.

\section{Klassifizierung von elastokapillaren Selbstorgani- sationsmethoden}

Die endgültige Ordnung wird durch die Methode beeinflusst, mit der die Filamente der Flüssigkeit ausgesetzt werden (d.h. den Benetzungs- und Trocknungsschritten). Im Folgenden diskutieren wir die wesentlichsten Methoden, die bis heute entwickelt wurden. Diese Methoden sind in Abbildung 2 zusammengefasst.

Eintauchen: Das einfachste und häufigste Verfahren der kapillaren Selbstorganisation ist das Eintauchen des Substrats in ein mit der Benetzungsflüssigkeit gefülltes Becherglas, ${ }^{[3,13,23,43,55,64-67]}$ wobei die Flüssigkeit typischerweise ein organisches Lösungsmittel ist. Anschließend wird das Substrat aus dem Becherglas gezogen, und die Flüssigkeit wird unter Umgebungsbedingungen verdampft. Dabei ist die Orientierung des Substrats offenbar nicht von Bedeutung, und im Allgemeinen ist davon auszugehen, dass es parallel zur Flüssigkeitsoberfläche ausgerichtet ist. Dieses Verfahren kann ohne Weiteres angewendet werden, um verschiedene Arten von Nanostäben und Nanoröhren zu separieren.

Gerichtetes Eintauchen: Beim gerichteten Eintauchen ist das Substrat in Bezug auf die Flüssigkeitsoberfläche speziell ausgerichtet, um die Richtung der auf die Strukturen ausgeübten Kapillarkräfte beim Kontakt mit der Flüssigkeitsoberfläche zu steuern. Genauer gesagt wurde diese Methode entwickelt, um die Orientierung von Nanofilamenten zu verändern, wenn sie aus der Flüssigkeit gezogen werden, ${ }^{[27]}$ was eine Verschiebung der Filamente von vertikal zu hori- 


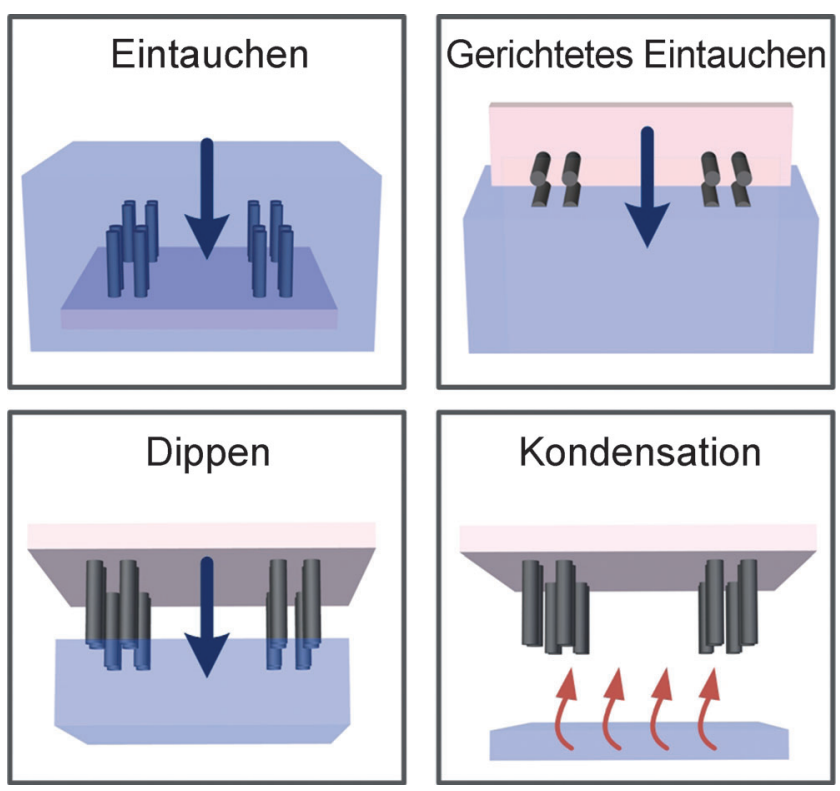

Abbildung 2. Übersicht über die zur elastokapillaren Selbstorganisation verwendeten Benetzungsmethoden.

zontal (also parallel zum Substrat) verursacht. ${ }^{[27,29]}$ Ein Beispiel ist die Faltung von vertikalen CNT-Mikrostrukturen zur Bildung horizontal angeordneter Filme. ${ }^{[27,29]}$

Dippen: Variationen des Eintauchverfahrens wurden entwickelt, damit die Kapillarkräfte stärker lokalisiert und mit gleichmäßiger Steuerung auf die Nanofilamente einwirken können. Beim Dip-Verfahren wird das Substrat parallel zur Flüssigkeitsoberfläche umgedreht und dann abgesenkt, bis die Spitzen der Nanofilamente die Flüssigkeitsoberfläche berühren. Dann wird die Flüssigkeit durch den Kapillareffekt in die Zwischenräume zwischen den Nanofilamenten gezogen. Da die Flüssigkeit lokal absorbiert wird, ${ }^{[17,65,68]}$ sollte dieses Verfahren prinzipiell die Gleichmäßigkeit einer Anordnung mit zusammentreffenden Spitzen unterstützen, wie sie im nächsten Abschnitt beschrieben wird.

Kondensation: Eine feinere Steuerung der Einführung einer benetzenden Flüssigkeit wird erreicht, wenn die Flüssigkeit auf das Substrat und die Nanofilamente kondensiert wird. Dies ist insbesondere bei der Verarbeitung fragiler Strukturen von Nutzen, die aufgrund der während des Eintauchens oder Dippens von der Flüssigkeitsoberfläche ausgeübten Kapillarkräfte beschädigt werden könnten (z.B. delaminiert, aggregiert oder unerwünscht reorientiert). Bei diesem Verfahren wird das Substrat über einem hohen Becherglas umgedreht, das eine geringe Menge einer heißen Flüssigkeit wie Aceton enthält, wie in Abbildung 2 dargestellt. ${ }^{[35]}$ Da das Substrat eine niedrigere Temperatur als die Flüssigkeit aufweist, kondensiert der Dampf auf dem Substrat, wodurch die Flüssigkeit in den Kapillaren von selbst in einzelnen Gruppen der Strukturen auf dem Substrat nach oben steigt. ${ }^{[19,24,69]}$

Außerdem sei darauf hingewiesen, dass einige dieser Benetzungsverfahren mit einer mechanischen Vorbehandlung kombiniert wurden, um zuerst die Reorganisation der vertikalen Nanofilamente zu dirigieren. Zum Beispiel wurden sie vor dem Eintauchen und Trocknen einer Scherung ${ }^{[20]}$ oder einem Walzen ${ }^{[70]}$ ausgesetzt. Ein weiteres Verfahren zum vorsichtigen Aufbringen von Flüssigkeit nutzt eine Blase, um die Spitzen von strukturierten CNT-Wäldern in Kontakt zu bringen, was eine lokale Verdichtung der oberen Bereiche des CNT-Waldes verursacht. ${ }^{[69]}$

\section{Klassifizierung von Oberflächenmustern nach elastokapillarer Selbstorganisation}

Um Regeln für ein Design der Oberfläche zu entwickeln, die sowohl Theorie als auch Wissen und Intuition berücksichtigen, ist eine Klassifizierung nach Geometrien hilfreich, die durch elastokapillare Selbstorganisation erreicht werden können. Um dies zu veranschaulichen, finden sich für jede Klassifizierung beispielhafte Abbildungen, unterteilt nach Anzahl von Säulen pro Cluster, $N=1-10, N=1-100$ und $N>100$. Im letzten Fall werden meistens Wälder aus verflochtenen Filamenten wie CNT verwendet. Die Beispiele sind in Abbildung 3 zusammengestellt und werden im Folgenden detailliert diskutiert.

Zusammentreffende Spitzen (,,Top-Gathering“): Zu den ersten eingehenderen Untersuchungen der kapillaren Selbstorganisation von Nanofilamenten gehört der Bericht von Tanaka et al. aus dem Jahr $1993 .{ }^{[15]}$ Sie benutzten strukturierte Polymernanosäulen mit einem Durchmesser von $200 \mathrm{~nm}$ und beobachteten die Bildung von pyramidalen „Hütten“ durch das Zusammentreffen der Spitzen dieser Säulen nach Abspülen und Trocknen der Entwicklungsflüssigkeit. In Abbildung 4 finden sich typische Beispiele von „Top-Gathering“-Säulen mit zunehmender Anzahl pro Anordnung. Wie bei Tanaka beschrieben, wird der Selbstorganisationsprozess stark vom Aspektverhältnis und dem Abstand der Nanofilamente beeinflusst [wie durch Gl. (1) und (2) erklärt]. Während die Nanofilamente im Idealfall zu perfekten, dicht gepackten Clustern aggregieren können, verhindert die Biegesteifigkeit der Filamente sowie Schwankungen des anfänglichen Abstands der Nanofilamente, Welligkeit oder gar Verflechtungen typischerweise eine perfekte Packung. ${ }^{[3,19,74]}$ So verstärkte die Verdichtung durch das Zusammentreffen der Spitzen von CNT-Wäldern z.B. deren Querschnittsdichte bei einem Tauchbeschichtungsverfahren um das 5- bis 25-fache, ${ }^{[68]}$ bei delaminierten SWNT-Wäldern mit einem Durchmesser von $2.8 \mathrm{~nm}$ um das 20 -fache ${ }^{[3]}$ und bei plasmabehandelten MWNT-Wäldern um das 30-fache (Abbildung $4 \mathrm{e}) .{ }^{[19]}$ Dies trifft jedoch typischerweise auf nicht mehr als $50 \%$ der Idealdichte von hexagonal gepackten CNTs zu.

Wicklung: Kang et al. haben beschrieben, wie ein $\mathrm{Zu}-$ sammenspiel aus Steifigkeit, Geometrie und Chemie von Nanofilamenten anstelle von Pyramiden aus zusammentreffenden Spitzen (Abbildung 4) eine Aggregation zu chiralen (verdrillten) Anordnungen verursacht (Abbildung 5). ${ }^{[6]}$ Der Übergang von einer die Top-Gathering-Anordnung zu einer sich chiral windenden Anordnung hängt von einem empfindlichen Gleichgewicht zwischen Adhäsion, Elastizität und Kapillarität ab. ${ }^{[56]}$ Zum Beispiel bildeten Polymernanosäulen mit starker gegenseitiger Adhäsion „Hütten“, während Na- 

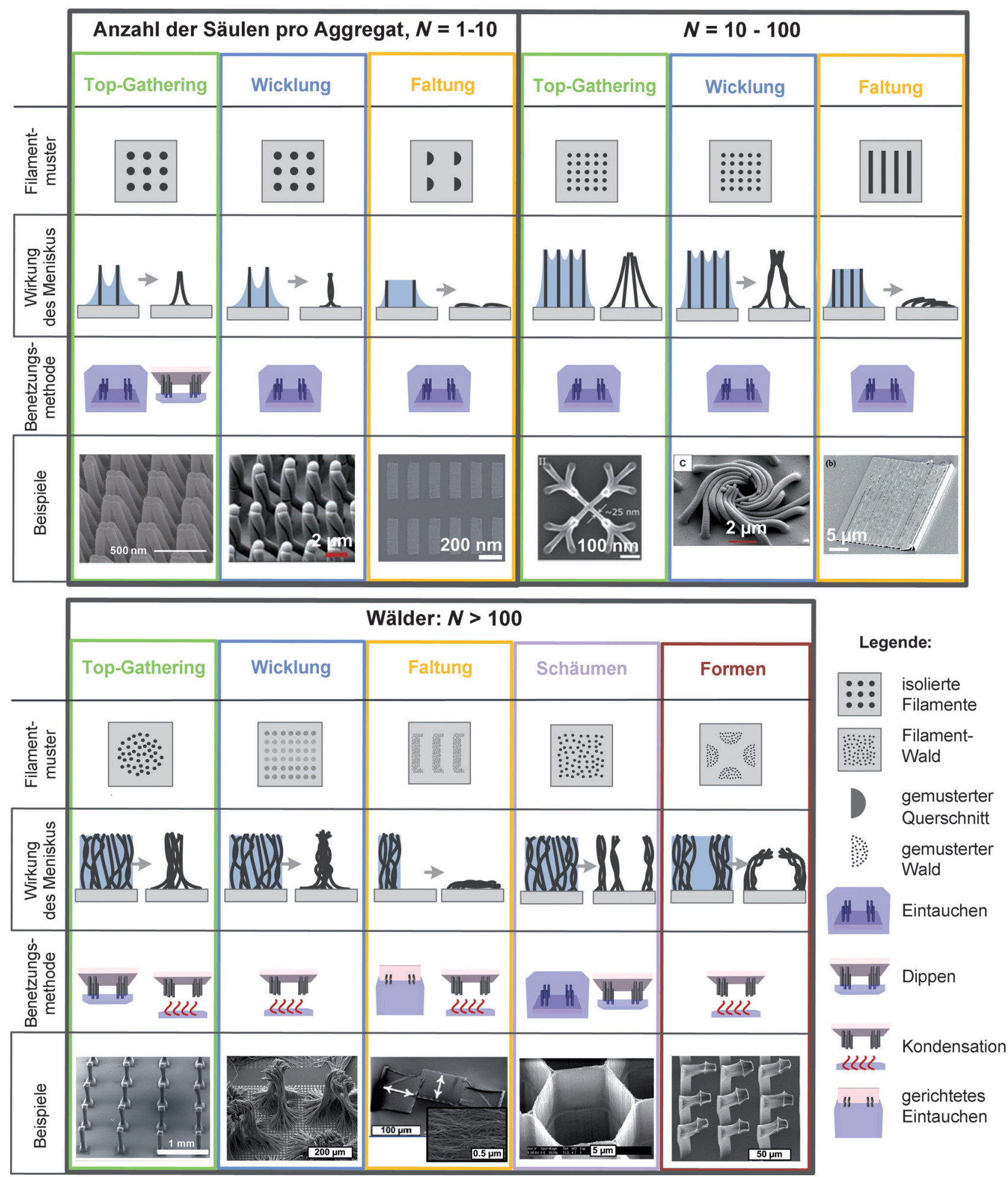

Abbildung 3. Klassifizierung von Oberflächendesigns, die durch elastokapillare Selbstorganisation erreicht werden, unterteilt nach Anzahl von $\mathrm{Na}$ nofilamenten pro Aggregat und der wesentlichen Aggregationsbewegung: Top-Gathering, Wicklung, Faltung, Formung und Schäumung. Von links nach rechts, Nachdruck mit Genehmigung aus Lit. [71] (Copyright 2011 American Chemical Society), Lit. [55] (Copyright AAAS), Lit. [44] (Copyright 2011 Wiley), Lit. [43] (Copyright 2010 American Chemical Society), Lit. [72] (Copyright 2011 IOP), Lit. [73] (Copyright 2011 IEEE), Lit. [26] (Copyright 2011 American Chemical Society).

nosäulen mit schwächerer Adhäsion, die ein Gleiten erlaubte, chirale Anordnungen bildeten. ${ }^{[56]}$ Nachdem die Säulen an den Spitzen miteinander in Kontakt gekommen sind, rutschte dieser Kontaktpunkt nach unten und die Säulen wickelten sich auf chirale Weise umeinander. Verstärken der Adhäsion durch eine Plasmabehandlung von Epoxidharz-Nanofila- 

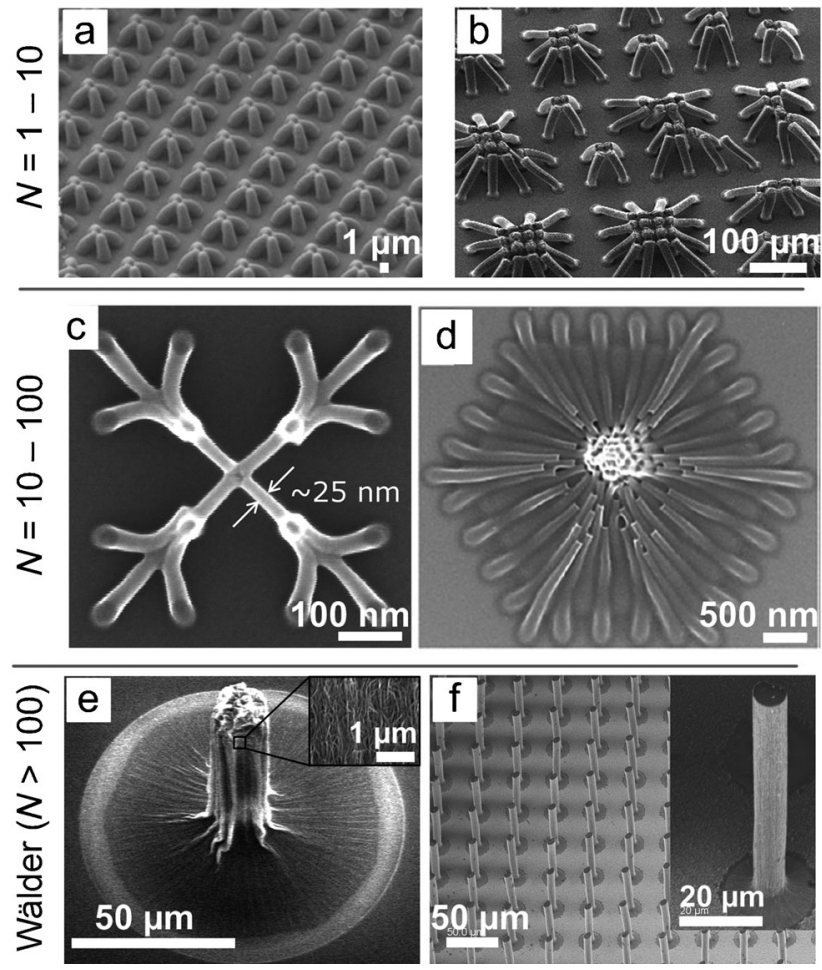

Abbildung 4. Beispiele für durch kapillare Selbstorganisation hergestellte „Top-Gathering“-Säulen. a) Durch Elektronenstrahllithographie hergestellte Photolacksäulen. Nachdruck mit Genehmigung aus Lit. [75], Copyright 2009, American Institute of Physics. b) SU8-Epoxidharz-Säulen nach kapillarer Selbstorganisation und nachfolgender Pyrolyse, ähnlich wie in Lit. [59]. c,d) Anordnungen von Polymernanosäulen nach Elektronenstrahllithographie und kapillarer Selbstorganisation zu Clustern. Nachdruck mit Genehmigung aus Lit. [43], Copyright 2010 American Chemical Society. e,f) Mikrosäulen aus CNT-Wäldern, die durch kapillare Selbstorganisation aggregieren. Nachdruck mit Genehmigung aus Lit. [19], Copyright 2011 IOP und 2006 Macmillan. ${ }^{[3]}$

menten verhinderte das Gleiten und hemmte demzufolge die Wicklung. Ebenso verhindern große steife Säulen eine chirale Wicklung, und zwar aufgrund der hohen elastischen Rückstellkraft. Zufällig haben wir eine geringfügige Wicklung nach einer kapillaren Selbstorganisation von CNT-Mikrosäulen mit hohem Aspektverhältnis beobachtet. Dies ist ein hierarchischer Prozess, bei dem jedes einzelne Säulenaggregat (wie in Abbildung 4e) und gleichzeitig Gruppen von Säulen zu den in Abbildung $5 \mathrm{f}$ dargestellten gewickelten Strukturen zusammenkommen.

Faltung: Während Top-Gathering und Wicklung durch Kapillarkräfte zwischen den Filamenten verursacht wird (d.h. verbrückende Menisken), können Kapillarkräfte zwischen den Filamenten und dem Substrat ein Fallen der Filamente auf das Substrat verursachen. Daher kann das Benetzen und Trocknen auch eine kontrollierte Faltung von Filamenten und dünnen vertikalen Strukturen ermöglichen. Beim Modellsystem eines isolierten Filaments tritt Reorientierung auf, wenn die durch den Benetzungsmeniskus ausgeübte vertikale Kraft die zur Krümmung der Struktur zum Substrat notwendige Kraft übersteigt. Eine Reorientierung kann auch auftreten, wenn der Meniskus die Struktur während des Verdampfens in Richtung des Substrats beugt. Auf jeden Fall
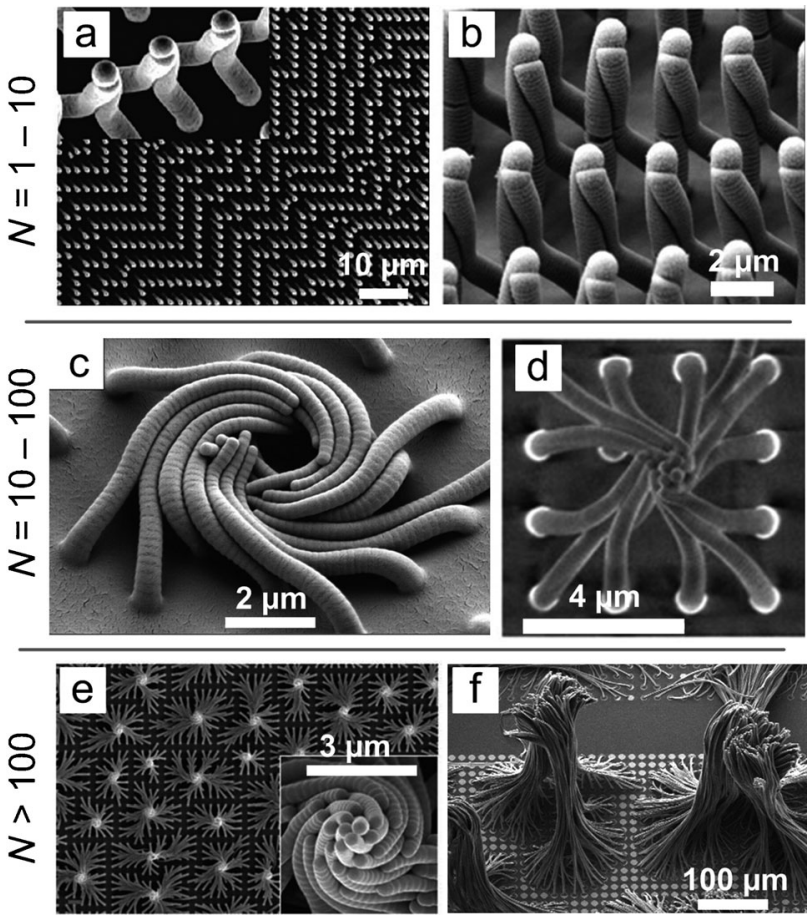

Abbildung 5. Gewickelte Anordnungen von Nanofilamenten als Funktion der Anzahl von Säulen pro Aggregat. a-e) Wickelung von Polymerfilamenten. a) Nachdruck mit Genehmigung aus Lit. [56], Copyright 2010 American Chemical Society. b-e) Nachdruck aus Lit. [55], Copyright AAAS. f) Wicklung von Kohlenstoffnanoröhren-Wäldern.

begünstigt ein reißverschlussartiges Ablösen des Meniskus zwischen Struktur und Substrat während der Verdampfung die Stabilität der gefalteten Konfiguration. ${ }^{[26]}$ Dieses Prinzip wurde angewendet, um Reihen aus asymmetrischen PMMABalken mit einer Breite von $10 \mathrm{~nm}$ (Abbildung 6a,b,d) ${ }^{[44]}$ sowie PMMA-Nanoblätter $\mathrm{zu}$ falten (Abbildung 6c). ${ }^{[72]}$ Mikrowände aus CNT-Wäldern wurden durch gerichtetes Eintauchen gefaltet (Abbildung 6e), ${ }^{[27,29]}$ was zu horizontal ausgerichteten (HA-)CNT-Filmen mit einem Packungsanteil von $42 \%$ führte. Dabei fallen alle CNT gesteuert durch die Eintauchrichtung in die gleiche Richtung. Später wurde gefunden, dass die CNT-Faltungsrichtung durch eine eingebaute Asymmetrie im Querschnitt des CNT-Waldes bestimmt werden kann, was die Herstellung von multidirektionalen HA-CNT-Mustern über große Flächen ermöglichte (Abbildung $6 \mathrm{f}){ }^{[26]}$

Schäumen: Werden Filme aus eng benachbarten, flexiblen Nanosäulen einer benetzenden Flüssigkeit ausgesetzt, bilden sich große Aggregate, die einem zweidimensionalen, offenzelligen Schaum ähneln. Dieses Verhalten wurde intensiv an CNT-Wäldern $^{[19,23,24,33,64,76]}$ sowie an Anordnungen von $\mathrm{Si}_{-},{ }^{[36,37,66]} \mathrm{Cu}^{-}{ }^{[66]}$ und $\mathrm{ZnO}-\mathrm{Nanodrähten}{ }^{[41]}$ untersucht. Bei CNT-Wäldern wirken während des Verdampfens der Flüssigkeit konkurrierende Kräfte, nämlich die Kontraktion des Waldes einerseits und die Steifigkeit der CNT und deren Adhäsion auf dem Substrat andererseits, was die Bildung von zufällig verteilten Hohlräumen innerhalb der fertigen Anordnung verursacht. Chakrapani et al. ${ }^{[23]}$ haben für CNTs beschrieben, dass die mittlere Hohlraumgröße je nach der Länge (Höhe) der CNTs im Wald linear variiert. 

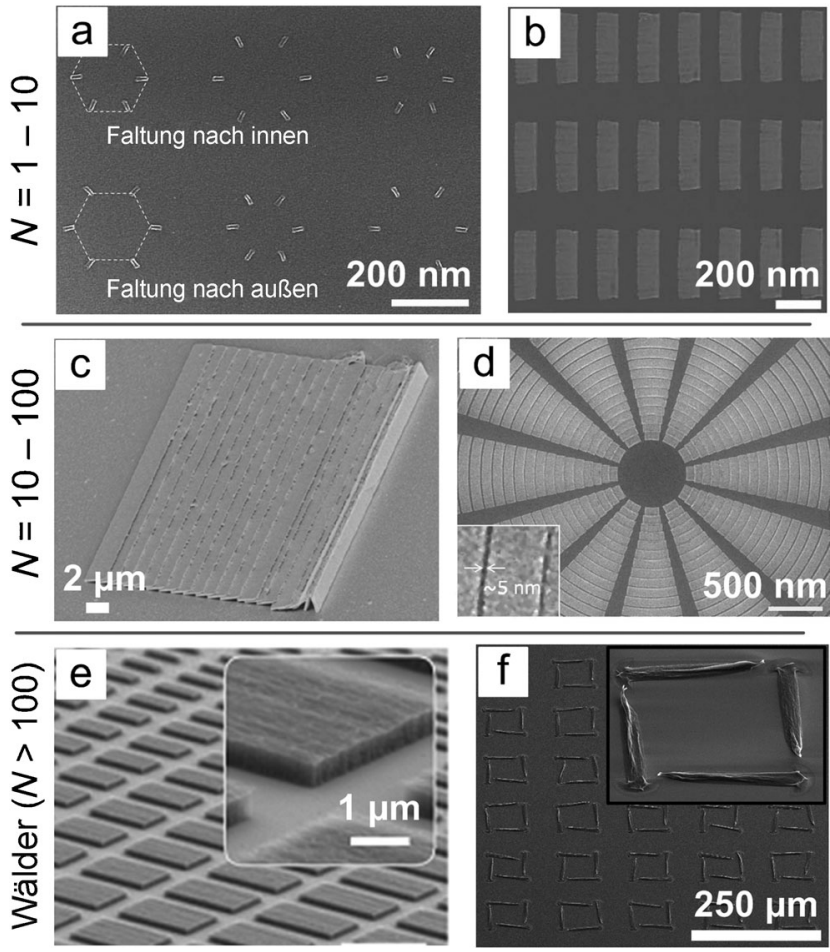

Abbildung 6. Elastokapillare Faltung von Nanosäulen als Funktion der Anzahl von Säulen pro Aggregat. a,b,d) Durch Eintauchverdichtung gefaltete Elektronenstrahl-Photolacklinien. Nachdruck mit Genehmigung aus Lit. [44], Copyright 2011 Wiley. c) Durch Eintauchverdichtung gefaltete PMMA-Blätter. Nachdruck mit Genehmigung aus Lit. [72], Copyright 2011 IOP. e) Durch gerichtetes Eintauchen gefalteter CNT-Wald. Nachdruck mit Genehmigung aus Lit. [27], Copyright 2008 Macmillan. f) Mittels Kondensation gefaltete, multidirektionale CNT-Wälder. Nachdruck mit Genehmigung aus Lit. [26], Copyright 2011 American Chemical Society.

Bei Modellsystemen kann die „Phasengrenze“ zwischen der Bildung von Hohlräumen und der Bildung von einzelnen Aggregaten in guter Übereinstimmung mit experimentellen Ergebnissen analytisch vorhergesagt werden. ${ }^{[19]}$ Auch numerische Modelle, die auf Netzwerken aus mechanischen Federn basieren, sagen die Verteilung und Geometrie der Hohlräume überraschend gut vorher. ${ }^{[76]}$

Während nicht-strukturierte CNT-Wälder zu zufällig verteilten Hohlräumen führen, kann deren Anordnung andererseits programmiert werden, indem vor der Selbstorganisation im Wald ein Lochmuster angelegt wird. Dies erfolgt durch eine Musterbildung auf dem Katalysator vor dem Züchten der CNTs oder mittels Ätzen des CNT-Walds nach dem Züchten (z.B. unter Verwendung von Laserablation). Die Löcher initiieren eine Hohlraumbildung und ermöglichen daher eine sehr einheitliche Anordnung von Zellen über große Bereiche. In Abhängigkeit vom Lochmuster werden hexagonale („bienenwabenartige“), quadratische oder andere Gitter erhalten, wie in Abbildung $7 \mathrm{c}, \mathrm{d}$ und in der Literatur dargestellt. ${ }^{[23,24,33,64]}$ Darüber hinaus sind CNTSchäume mechanisch robust und können direkt vom Substrat abgezogen oder durch Eintauchen in verdünnte, wässrige Flusssäure abgetrennt werden.
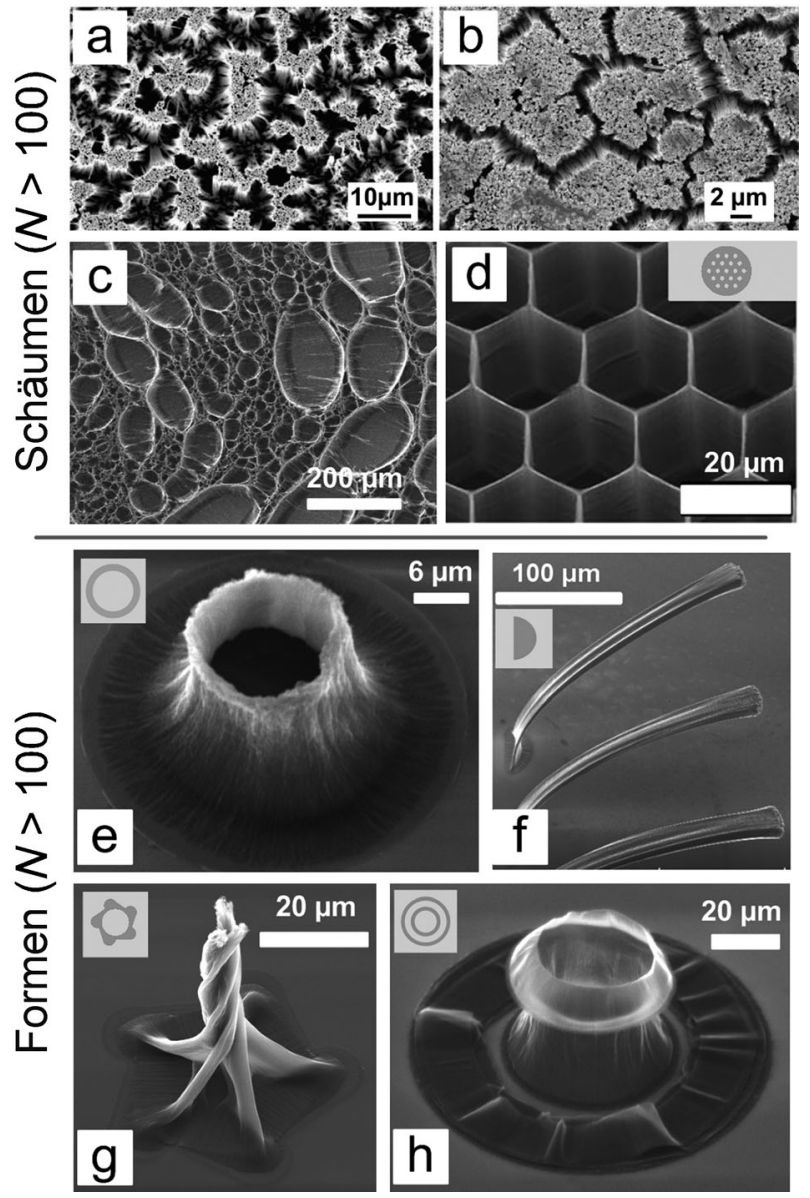

Abbildung 7. Beispiele für elastokapillare Selbstorganisation über Schäumen und Formen. a) Elastokapillares Schäumen eines Si-Nanodraht-Waldes. b) Elastokapillares Schäumen eines Cu-NanodrahtWaldes. Nachdruck mit Genehmigung aus Lit. [38], Copyright 2009 American Chemical Society. ${ }^{[38]}$ c) Schäumen von ungemusterten CNTWäldern unter Verwendung der Eintauchverdichtung. d) Schäumen von strukturierten CNT-Wäldern mittels Kondensationsverdichtung in einem Templat. Nachdruck mit Genehmigung aus Lit. [24], Copyright 2010 Wiley. e) Formen eines zylindrischen CNT-Walds zu einem Kegel (anfänglicher Querschnitt im Einschub) ${ }^{[33]} \mathrm{f}$ ) Kapillares Formen von CNT-Wäldern zu gebogenen Säulen mittels Kondensation. Nachdruck mit Genehmigung aus Lit. [73], Copyright 2011 IEEE. g) Kapillares Formen von helikalen CNT-Mikrosäulen mittels Kondensation; Herstellungsverfahren ähnlich wie in Lit. [77]. h) Herstellung von rückspringenden Strukturen durch wiederholtes kapillares Formen und Züchten von CNTs; Herstellungsverfahren ähnlich wie in Lit. [33].

Formen: Innerhalb der dimensionalen Grenzen, die von diskreten, festen Aggregaten bei einer Selbstorganisation vorgegeben werden (d.h. nicht unter Schäumbedingungen), können verschiedenste komplexe 3D-Filamentaggregate entstehen. ${ }^{[24]}$ Zum Beispiel ergab die Kondensationsverdichtung von dünnwandigen Zylindern ausgehend von CNT-Mikrowäldern, die aus einem lithographisch mit einem Muster versehenen Katalysator gezüchtet wurden, bei Aggregation eine Umwandlung zu abgeschrägten Mikroschächten (Abbildung 7e) und verursachte bei halbzylindrischen Säulen eine seitliche Auslenkung (Abbildung 7 f). Es können auch komplexere Geometrien programmiert werden, wie die Bil- 
dung von Helizes mit festgelegter Händigkeit (Abbildung $7 \mathrm{~g}){ }^{[32]}$ In diesen Beispielen definiert die anfängliche Geometrie des CNT-Walds zusammen mit der Dichte und Anordnung der CNTs innerhalb des Walds die Endgeometrie der Strukturen. ${ }^{[24,78]}$

Des Weiteren kann die kapillare „Formung“ von CNTs mehrfach wiederholt werden, um komplexe, geriffelte Mikrostrukturen mit sowohl symmetrischen (Abbildung 7h) als auch asymmetrischen Formen zu schaffen (siehe „Formen“ in Abbildung 3). ${ }^{[33,79]}$ Analytische Modelle ${ }^{[78]}$ und Finite-Element-Modelle ${ }^{[73]}$ können die grundlegenden Formumwandlungen vorhersagen, die bei einer kapillaren Formung auftreten. Im Zusammenhang mit den zu erwartenden Verbesserungen bei der Züchtung und Charakterisierung von CNTs bietet sich hier ein zuverlässiger Weg, um die kapillare Formung künftig zum Aufbau großflächiger 3D-mikrostrukturierter Oberflächen mittels CNTs zu verwenden.

\section{Prozesssteuerung}

Es hat sich gezeigt, dass abgesehen vom Material, der Geometrie und Organisation der Filamente auch andere Herstellungsschritte und Prozessmodifizierungen einen Einfluss auf den Selbstorganisationsprozess ausüben und zusätzliche Funktionalitäten auf den endgültigen Oberflächen ermöglichen. Dieser Abschnitt bietet eine kurze Übersicht über diese Methoden.

Oberflächenchemie: Die Oberflächenchemie der Filamente beeinflusst die Stärke und Stabilität ihrer Wechselwirkungen während der Selbstorganisation. Dies wurde untersucht, indem Epoxidharz-Nanosäulen mit einer dünnen Goldschicht beschichtet und dann selbstorganisierte Monolagen (SAM) aus Alkanthiol auf die Oberfläche aufgebracht wurden (Abbildung 8a). Es zeigte sich, dass der Prozentsatz stabiler Cluster bei kurzkettigen Molekülen von der Stärke der zu erwartenden chemischen Bindungen zwischen den jeweiligen funktionellen Gruppen abhängt. Des Weiteren ergeben SAMs mit längeren Kohlenstoffketten geordnetere Monolagen und daher gewöhnlich stabilere Cluster. ${ }^{[16]}$ Diese Oberflächenwechselwirkungen ermöglichen eine Steuerung der Reversibilität der Anordnung. Zum Beispiel erlaubt eine Verringerung der Adhäsion zwischen den Filamenten beim Verdampfen der Flüssigkeit ein Rückspringen der Filamente. ${ }^{[16]}$

Flüssigkeit und Verdampfungsmethode: Grundsätzlich kann jede Flüssigkeit, die die Filamente und das Substrat benetzt, für die elastokapillare Selbstorganisation verwendet werden. ${ }^{[15]}$ Der Einfluss der Flüssigkeitseigenschaften, wie z. B. Oberflächenspannung, ${ }^{[19]}$ Viskosität und Dynamik beim Benetzen und Trocknen (z.B. die Verdampfungsgeschwindigkeit), wird jedoch noch weiter untersucht. So berichteten z.B. Chakrapani et al. über die Verdichtung von CNT-Wäldern zu zellulären Schäumen unter Verwendung von Aceton, Toluol, Dimethylformamid, Tetrahydrofuran und Methanol. ${ }^{[23]}$ Futuba et al. berichteten über eine CNT-Verdichtung unter Verwendung von Wasser, Alkoholen, Aceton, Hexan, Cyclohexan, Dimethylformamid, flüssigem Stickstoff, Dioctylether, Ölsäure und Maschinenölen. ${ }^{[3]}$
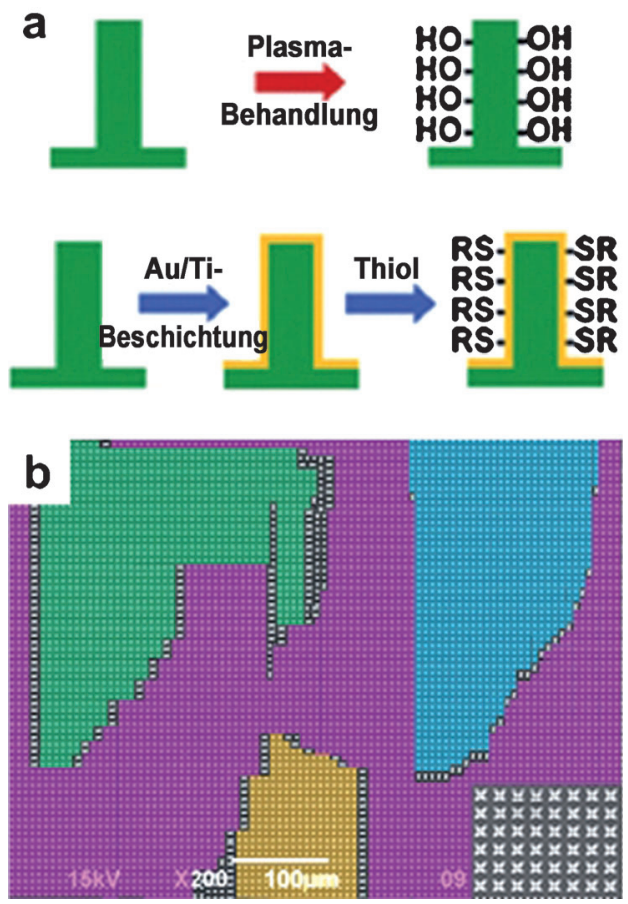

Abbildung 8. a) Methoden zur Veränderung der Oberflächenchemie von Nanofilamenten. b) Phasendiagramm von Einzeldomänenregionen mit elastokapillaren Aggregaten aus Polymernanofilamenten. Nachdruck mit Genehmigung aus Lit. [16], Copyright 2012 Elsevier.

Bei Polymernanosäulenanordnungen wurde gefunden, dass die Steuerung der Verdampfungsgeschwindigkeit eine bessere Einheitlichkeit des Prozesses ermöglicht. ${ }^{[16]}$ Abbildung $8 \mathrm{~b}$ zeigt Domänen, innerhalb derer die an der Spitze zusammentreffenden Säulen gleichförmig aggregieren. Darüber hinaus wird von einigen Forschern die Ansicht vertreten, dass eine Selbstorganisation erst dann auftritt, wenn sich die Flüssigkeit beim Verdampfen von den Nanodrähten zurückzieht, während andere meinen, dass die Aggregation sowohl während des Benetzens als auch des Trocknens auftritt. Erstere Ansicht wurde an verschiedenen Materialsystemen verifiziert, wobei Gefriertrocknung genutzt wurde ${ }^{[12,23]}$ und Säulen im nassen Zustand mittels AFM untersucht wurden. ${ }^{[15]}$ Futuba et al. berichteten über einen 20-\%igen Anstieg der Packungsdichte beim Eintauchen von CNT-Wäldern und eine signifikant stärkere Verdichtung beim Trocknen. ${ }^{[3]}$

Prozessgrenzen und Instabilitäten: Komplizierte und gleichförmige Filamentanordnungen finden sich typischerweise nur in einem kleinen Fenster des Parameterraums. Ebenso wie bestimmte Bedingungen erfüllt sein müssen, damit organisierte Strukturen stabil sind, ergeben sich drastisch unterschiedliche Ergebnisse, je nachdem ob beispielsweise die durch die Flüssigkeit ausgeübten Kräfte die Haftfestigkeit der Filamente auf dem Substrat übersteigen oder eine Faltung der Filamente auf das Substrat verursachen, anstatt die vertikalen Aggregate zu stabilisieren. Diese Einflüsse lassen sich zum Teil mithilfe der Theorie verstehen, genauso oft aber nur durch Empirie.

Zum Beispiel kann die vorher beschriebene, einfache Aggregationstheorie zur Vorhersage verwendet werden, ob 
a)
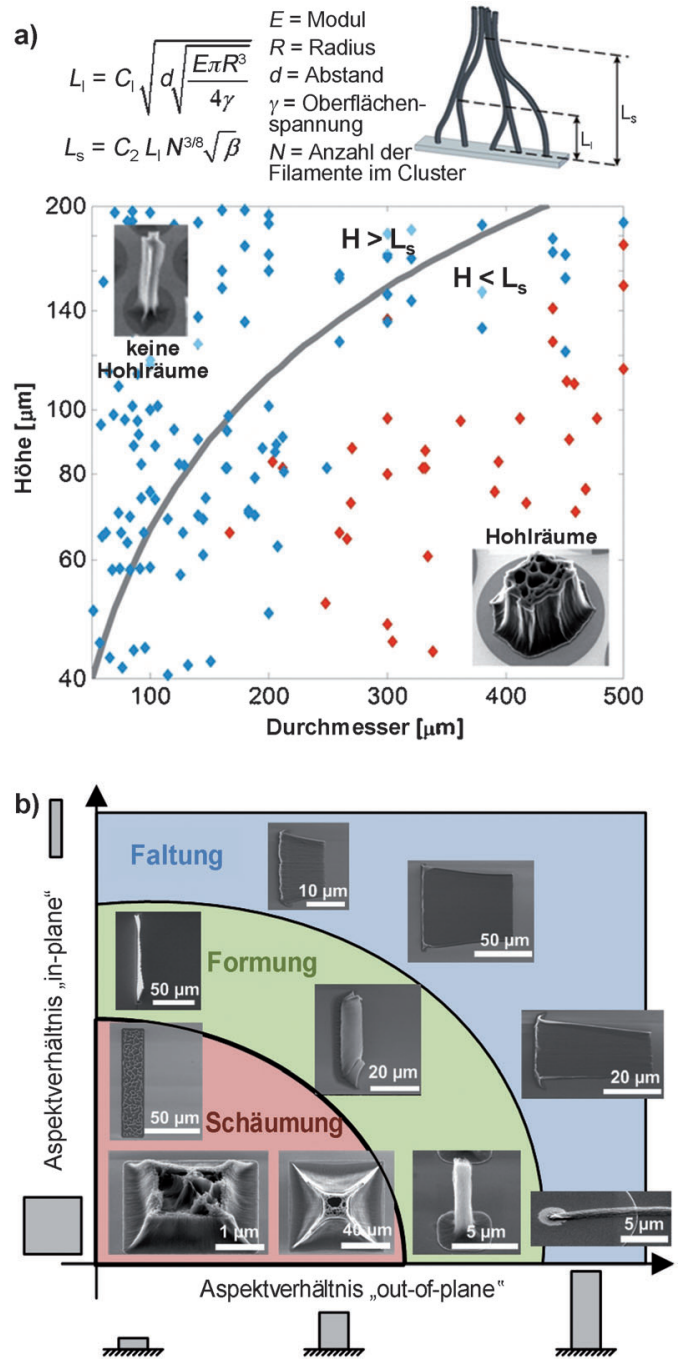

Abbildung 9. Prozesseinschränkungen bei der elastokapillaren Selbstorganisation von CNT-Wäldern. a) Theoretische und experimentelle Studien des Übergangs zwischen Schäumen und Formen von isolierten CNT-Mikrosäulen als Funktion der Höhe und des Durchmessers; veränderter Nachdruck aus Lit. [19]. b) Abbildung der Übergänge zwischen Schäumen, Formen und Falten in Bezug auf das Aspektverhältnis von CNT-Mikrosäulen in der Ebene und aus der Ebene heraus.

CNT-Mikrosäulen nach der kapillaren Selbstorganisation innere Hohlräume aufweisen oder zu einzelnen, festen Aggregaten verdichtet werden (Abbildung 9a). ${ }^{[19]}$ Am anderen Ende, d.h. bei Strukturen mit hohem Aspektverhältnis, wie z.B. schlanken, hohen CNT-Mikrosäulen oder dünnen, mit Mustern versehenen Wänden, verursachen die durch den benetzenden Meniskus ausgeübten, äußeren Kapillarkräfte eine Faltung der CNTs auf das Substrat. ${ }^{[2]}$ Abbildung 9b veranschaulicht, wie die elastokapillare Aggregation der CNT-Wälder als Funktion der anfänglichen Waldgeometrie vom Schäumen zum Formen und Falten übergeht.

Einfluss äußerer Beschränkungen: Das Aggregationsmuster kann auch beeinflusst werden, indem der Flüssigkeitsstrom mithilfe eines äußeren Templats gesteuert wird. Dies wurde unter Verwendung von Bienenwabenstrukturen demonstriert, um die Verdichtung der Polymernanosäulen auf kleinere Abschnitte zu begrenzen und die kapillare Selbstorganisation zu periodischen Mustern auf großen Flächen zu dirigieren (Abbildung 10a). ${ }^{[16]}$ Dabei wurde auch beobachtet, dass die kapillare Selbstorganisation von einander gegenüberliegenden Polymernanosäulen-Anordnungen zur Bildung von periodischen, chiralen Strukturen führen kann (Abbildung 10b). ${ }^{[80]}$
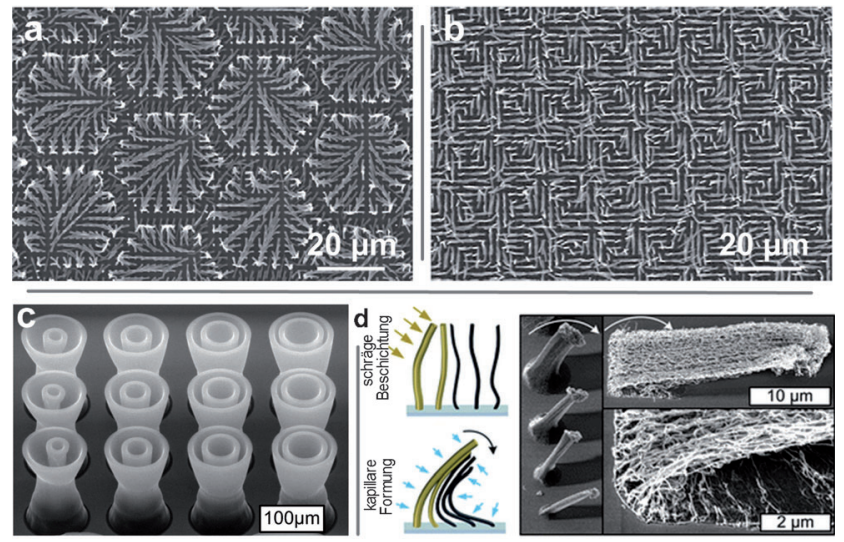

Abbildung 10. Darstellung von Verfahren zur Steuerung des elastokapillaren Prozesses. a) Aggregation mit einem hexagonalen Gitter als Templat. Nachdruck mit Genehmigung aus Lit. [16], Copyright 2012 Elsevier. b) Zu Moiré-Mustern selbstorganisierte Nanofilamente durch Verdampfen einer zwischen zwei Oberflächen mit periodischen Fasern eingeschlossenen Flüssigkeit. ${ }^{[16]} \mathrm{c}$ ) Partiell aggregierte CNT-Wälder. d) Kapillare Biegung von Janus-CNT-Wäldern. Nachdruck mit Genehmigung aus Lit. [28], Copyright 2012 Royal Society of Chemistry.

Unveröffentlichte Ergebnisse der Verdichtung von CNTWäldern haben gezeigt, dass es durch die Anwendung von winzigen Mengen Verdichtungsflüssigkeit möglich wird, die elastokapillare Aggregation beispielsweise nur auf den unteren Bereich eines CNT-Waldes zu beschränken (Abbildung 10c). Dies ermöglicht die Herstellung von rückspringenden Strukturen mit einem Gradienten in den mechanischen Eigenschaften. Des Weiteren kann die elastokapillare Aggregation ausgehend von CNT-Wäldern, die anisotrop durch eine schräge Metallverdampfung nur auf einer Seite beschichtet wurden, zu einer anisotropen Säulenbewegung führen, einschließlich einem Biegen der Säulen wie in Abbildung $10 \mathrm{~d}$ gezeigt. ${ }^{[28]}$ Dieser Ansatz ermöglicht eine feine Steuerung des Biegungswinkels und das horizontale Falten der Säulen.

\section{Anwendungen}

In diesem Abschnitt stellen wir ausgewählte Anwendungen von Oberflächen und Bauelementen vor, deren Funktionalität durch den Einsatz von elastokapillarer Selbstorganisation als Teil der Herstellung verbessert wurde. Viele dieser Anwendungen nutzen die bereits gut untersuchten CNTs (Abbildung 11). Mit zunehmender Zahl von Methoden zur Herstellung und Modifizierung anderer nanoskaliger Filamente wird sich dieser Bereich zukünftig zweifellos auf andere Materialien ausweiten (Abbildung 12). 
a)

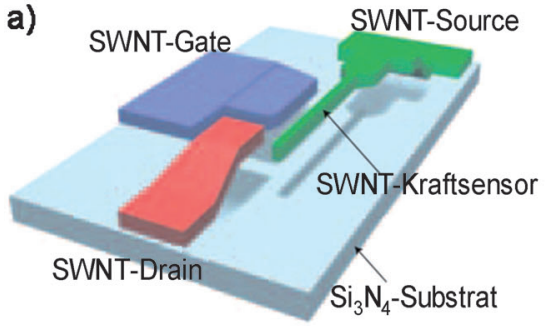

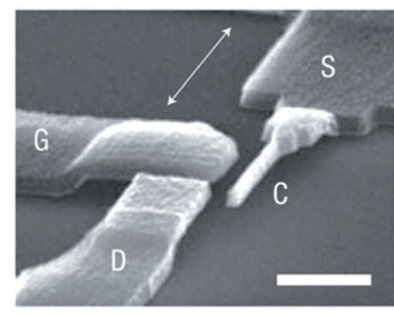

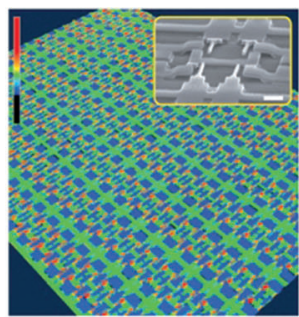

b)

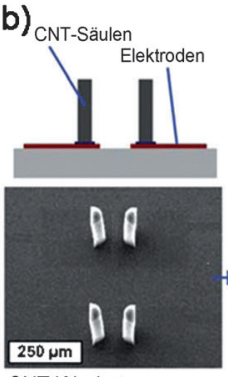

CNT-Wachstum
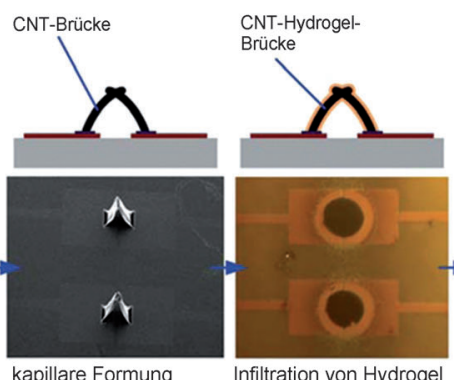

Infiltration von Hydroge

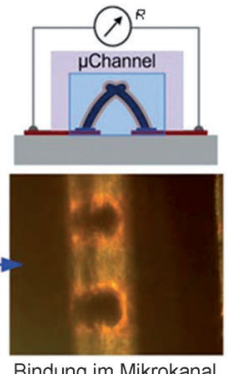

Bindung im Mikrokanal

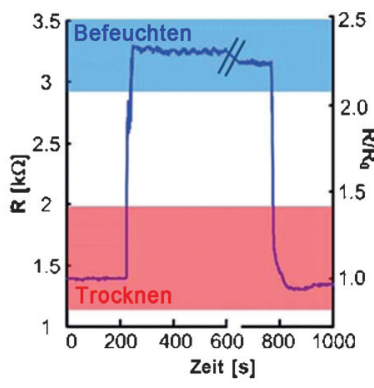

Zeit [s]
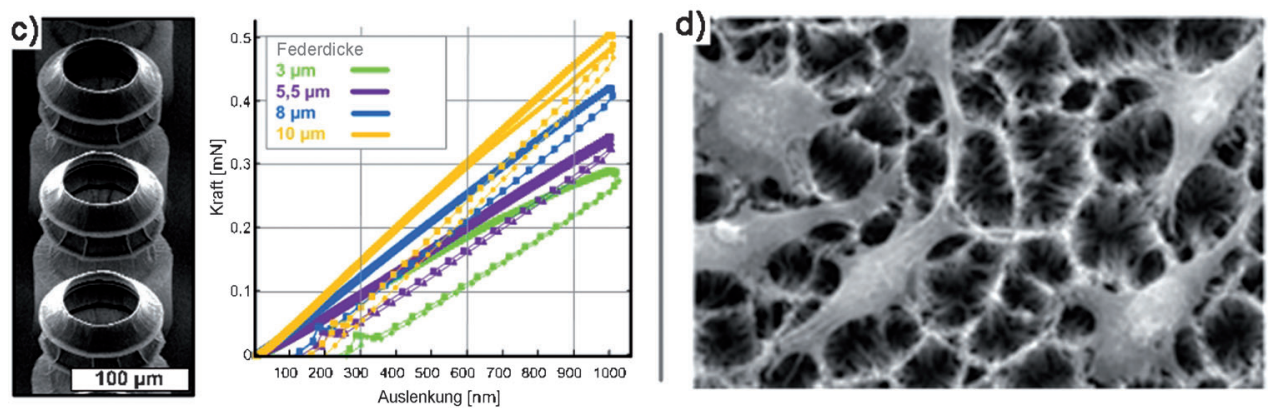
Auslenkung [nm]

e)

CNT-Wald Platzieren des auf $\mathrm{Si}$

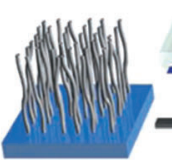
zwischen Objektträgern
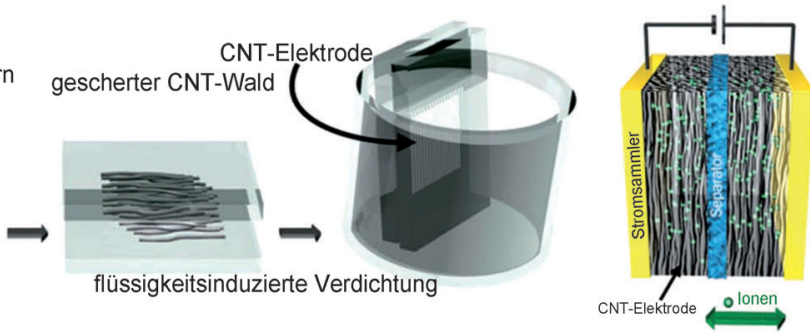

Abbildung 11. Anwendungen von elastokapillar selbstorganisierten CNTs. a) Mikrorelais aus horizontalen CNTs, die durch gerichtetes Eintauchen aggregiert wurden. Nachdruck mit Genehmigung aus Lit. [27], Copyright 2008 Macmillan. b) Hygroskopische Mikrosensoren, die durch kapillares Formen von 3D-CNT-HydrogelSensorknoten hergestellt wurden. Nachdruck mit Genehmigung aus Lit. [83], Copyright 2011 Royal Chemical Society. c) Durch wiederholtes Züchten und kapillares Formen von CNTs hergestellte, geriffelte CNT-Mikroblasebälge. Nachdruck mit Genehmigung aus Lit. [33], Copyright 2011 American Chemical Society. d) Schäumen von CNT-Zellen für die Zellzüchtung. Nachdruck mit Genehmigung aus Lit. [13], Copyright 2004 American Chemical Society. e) Mittels Scherung und Eintauchverdichtung von CNT-Wäldern hergestellter Superkondensator. Nachdruck mit Genehmigung aus Lit. [2], Copyright 2010 Wiley.

bisher erreichte Dichte von SWNTs ohne Nachbehandlung ist um einen Faktor 30 kleiner als dieses Ziel. ${ }^{[74]}$

Daher stellt die kapillare Selbstorganisation eine attraktive Methode dar, um CNT-Wälder mit geringer Dichte so zu behandeln, dass sie verdichtete Aggregate mit hoher Leitfähigkeit bilden. ${ }^{[17,82]}$ Liu et al. ${ }^{[17]}$ haben eine gründliche Untersuchung der elektrischen Leitfähigkeit von CNTSäulen vor und nach der kapillaren Selbstorganisation durchgeführt, die zeigte, dass die Selbstorganisation den Widerstand bedingt durch die verringerte Querschnittsfläche um das 15-fache verringerte. Lim et al. ${ }^{[65]}$ haben eine 3.5fache Abnahme des Widerstands nach Verdichtung gemessen. Besonders interessant ist, dass bei der Züchtung der CNT-Mikrosäulen auf TiN-Elektroden die nachfolgende kapillare Verdichtung auch den Kontaktwiderstand zwischen den CNTs und TiN verringerte, indem die Basis der Säulen zum Substrat geknickt wurde, während der obere Teil vertikal blieb. $^{[19]}$

\section{MEMS-Bauelemente} und Mikrosensoren: Kapillare Selbstorganisation ist ein attraktiver Weg, um funktionale, mechanische Komponenten unter Verwendung von dicht gepackten Nanodrähten und Nanoröhren mit kontrollierten Orientierungen aufzubauen. Zum Beispiel haben Hayamizu et al. nanome-

Mikroelektronische Durchkontaktierungen und Schaltkreise: Die hohe Strombelastbarkeit von CNTs, die bis zu $10^{9} \mathrm{~A} \mathrm{~cm}^{-2}$ beträgt, deutet darauf hin, dass diese $\mathrm{Cu}$ in mikroelektronischen Schaltkreisen ersetzen könnten. ${ }^{[81]}$ Die am besten etablierte Methode zur Einbindung von CNTs in der Mikroelektronik ist die direkte Erzeugung mittels CVD. ${ }^{[1,62]}$ Allerdings ergibt dieser Prozess häufig unzureichend geringe Packungsdichten von $1-5 \% .{ }^{[68]}$ Insbesondere die höchste chanische Bauelemente vorgestellt, die auf horizontal ausgerichteten CNT-Filmen basierten, die mittels kapillarer Selbstorganisation durch gerichtetes Eintauchen von strukturierten, vertikalen CNTs gebildet wurden. ${ }^{[27]}$ SWNT-Balken mit einem Querschnitt von nur $40 \times 70 \mathrm{~nm}$ (Breite $\times$ Höhe) wurden beispielsweise mittels Elektronenstrahllithographie mit einem Muster versehen. Jeder Balken bestand aus nur etwa 200 SWNTs, und daraus wurden 1276 elektrostatisch 

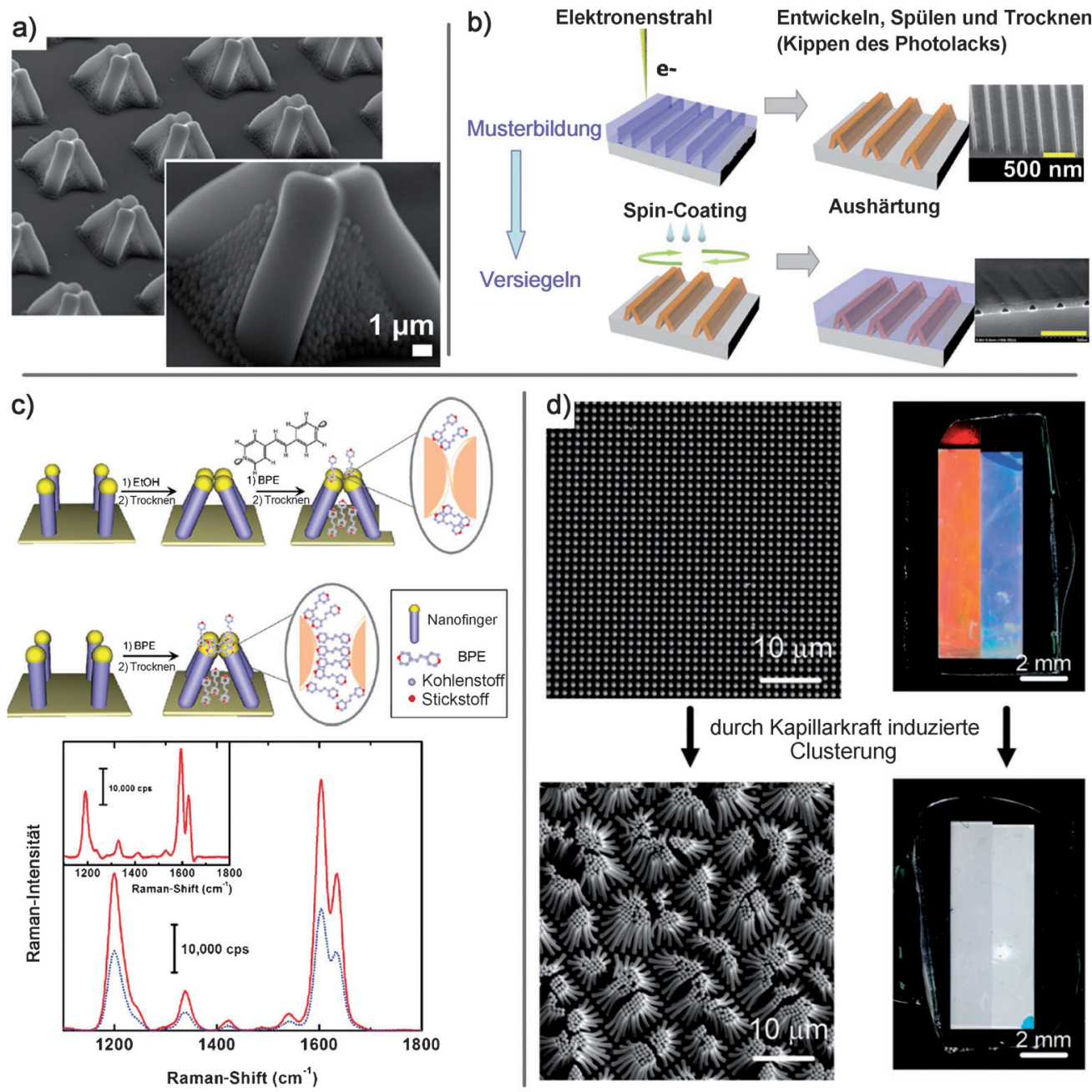

e)
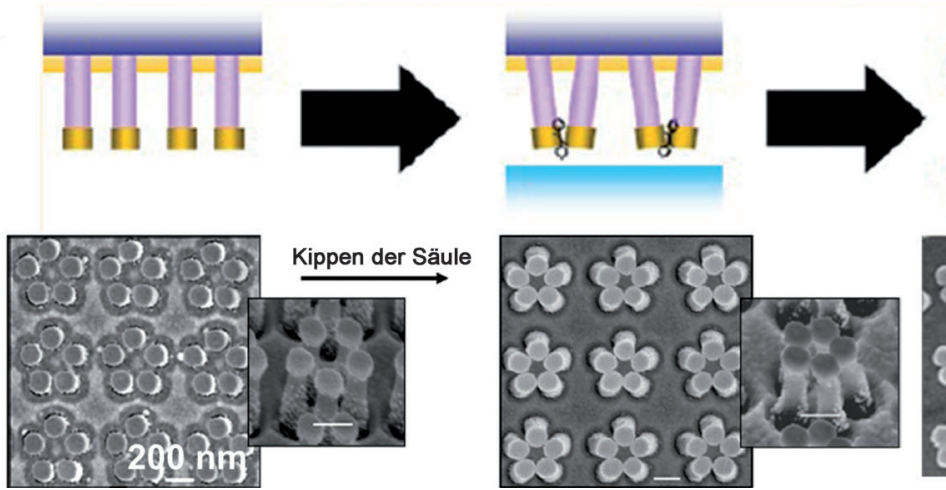

Abbildung 12. Anwendungen von elastokapillar aggregierten Polymernanofilamenten. a) Einschluss von Nanopartikeln unter Top-Gathering-Säulen. Nachdruck mit Genehmigung aus Lit. [87], Copyright 2008 Elsevier. b) Herstellung von nanoskaligen 3D-Kanälen durch Aggregation von Elektronenstrahl-Nanoblättern. Nachdruck mit Genehmigung aus Lit. [47]. Copyright 2008 American Institute of Physics. c) Einfang von Molekülen in TopGathering-Säulen und Vergleich von Raman-Spektren mit gefangenem Molekül (der Einschub zeigt den Beitrag der zwischen den Spitzen gefangenen Moleküle. Nachdruck mit Genehmigung aus Lit. [71]. Copyright 2011 American Chemical Society. d) Elastokapillare Modulation der Bragg-Beugungsfarbe in Hydrogel-Filamenten. Nachdruck mit Genehmigung aus Lit. [42]. Copyright 2009 American Chemical Society. e) Einfangen von Molekülen an Metallnanopartikeln und deren Transferdruck. Nachdruck mit Genehmigung aus Lit. [91]. Copyright 2012 American Chemical Society.

gesteuerte Relais-Bauelemente mit einer Ausbeute von 95.5\% hergestellt (Abbildung 11a). Die gleiche Gruppe be-
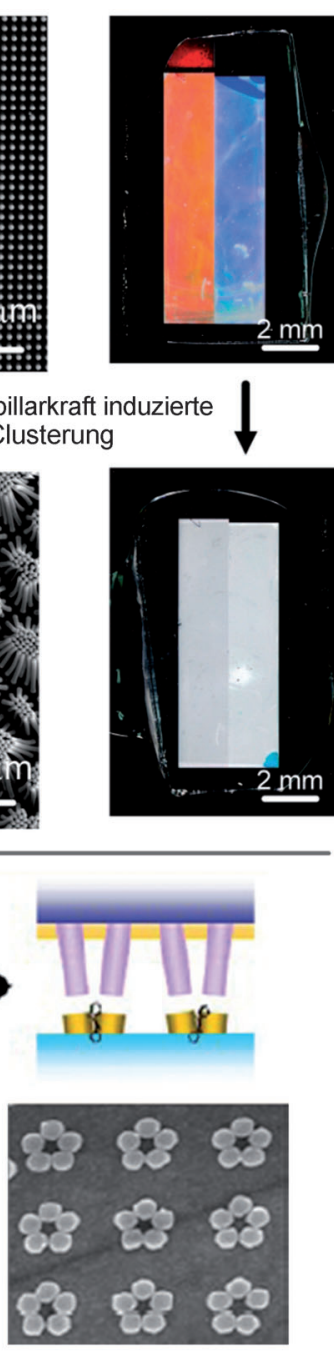

richtete auch über die Herstellung von freistehenden, dicht gepackten CNT-Balken als mechanische Resonatoren. ${ }^{[29]}$

CNT-Wälder wurden kapillar zu 3D-Brücken geformt, die mit Hydrogelen infiltriert und in Mikrokanäle eingebaut wurden, um hygroskopische Sensoren zu bilden (Abbildung 11b). ${ }^{[83,84]}$

Desgleichen wurden vertikale CNT-Schichten mittels kapillarem Falten transformiert und verdichtet, um Paare von strukturierten Elektroden auf dem Substrat zu verbinden. Die horizontal ausgerichteten CNTs erleichterten dann eine gerichtete Kristallisation von $\mathrm{C}_{60}$ aus der Lösung, um eine kombinierte hierarchische Struktur mit starkem Ansprechverhalten auf UVStrahlung $\mathrm{zu}$ ergeben. ${ }^{[70]}$ CNT-,,Mikroschächte“, die durch wiederholtes Züchten und kapillares Formen von CNTs hergestellt wurden, zeigten eine abstimmbare mechanische Verformbarkeit, was für kompakte vertikal Mikrofedern in Nadelkartenanordnungen nützlich sein könnte (Abbildung 11c). Durch eine Kombination aus Top-Down-Lithographie und Bottom-upSelbstorganisation entwickelten Choi et al. dreieckige Nanokanäle mit einer Breite von $30 \mathrm{~nm}$ und einer Länge von $10 \mu \mathrm{m}$ durch den kapillaren Kollaps von mittels Elektronenstrahl definierten Photolackwällen dung 12b). ${ }^{[4]}$

(Abbil-

Fallen und plasmonische Sensoren: Segawa et al. haben beobachtet, dass an der Spitze zusammentreffende Säulen Partikelansammlungen einfangen und organisieren können, wenn die Lösung z. B. $\mathrm{SiO}_{2}$ - und $\mathrm{ZnO}$ - 
Nanoperlen enthält (siehe Abbildung 12a). Die eingefangenen ZnO-Partikel zeigten eine starke Photolumineszenz. Zudem wurde gefunden, dass Top-Gathering-Anordnungen zur Herstellung neuer periodischer Muster für LumineszenzBauelemente von Nutzen sind. ${ }^{[85-89]}$ In diesem Fall sind die eingefangenen Partikel viel kleiner als die an der Spitze zusammentreffenden Säulen. Später zeigten Pokroy ${ }^{[55]}$ und Chen, ${ }^{[46]}$ dass auch Perlen gefangen werden können, die viel größer sind als einzelne Filamente. Die Autoren wiesen darauf hin, dass eine kontrollierte Partikelfreisetzung aus den Nanosäulen-Anordnungen auf Oberflächen für den Wirkstofftransport von Interesse sein könnte.

$\mathrm{Hu}$ et al. stellten in einem sehr viel kleineren Maßstab eine Molekülfalle auf der Basis von Top-Gathering-Säulen vor. Beim Aggregieren bildeten goldbeschichtete Nanofilamente „Hotspots“ für oberflächenverstärkte Raman-Spektroskopie (SERS) und Röntgenphotoelektronenspektroskopie (XPS). Dies wurde genutzt, um trans-1,2-Bis(4-pyridyl)ethylen einzufangen und $\mathrm{zu}$ identifizieren (Abbildung 12c). ${ }^{[71,90]}$ Des Weiteren wurden plasmonische Strukturen für SERS entwickelt, indem durch elastokapillare Selbstorganisation angeordnete Metallnanopartikel übertragen wurden, wie in Abbildung 12e dargestellt. ${ }^{[11,91]}$ Ein Vorteil dieses Verfahrens ist, dass Moleküle zwischen den Nanopartikeln gefangen und dann in nächster Nachbarschaft zueinander angeordnet werden können (enger als durch Lithographie möglich wäre).

Biosonden und Gerüste: Verglichen mit herkömmlichen flachen Metallelektroden bieten Kohlenstoffnanoröhren und Kohlenstoffnanofasern (CNFs) eine beträchtliche Vergrößerung der Oberfläche sowie eine gute elektrochemische Stabilität. Da diese Sonden in nassen Umgebungen arbeiten, sind Überlegungen zur Nutzung von kapillarer Selbstorganisation wichtig. So untersuchten Nguyen-Vu et al. den Einfluss von kapillarer Selbstorganisation auf CNFs an neuralen Mikroelektrodenarrays. ${ }^{[12]}$ Sie beobachteten, dass die Spitzen von CNF-Mikrobündeln mit Top-Gathering-Struktur den Zellkörper aufspießen oder die Zellmembran ähnlich wie eine Zeltstange hochdrücken können. Kleinere Zellkörper überspannen den offenen Raum und sind zwischen den Spitzen von benachbarten Mikrobündeln aufgehängt, während andere den Kontakt mit den Nachbarzellen verlieren und in das Tal fallen. Diese an der Spitze zusammentreffende Topographie ergibt einen vollständig anderen Adhäsionsmechanismus als ähnliche, mit Polypyrrol (PPy) beschichtete CNF-Röhren, die aufgrund der erhöhten mechanischen Steifigkeit keine kapillare Selbstorganisation zeigten. Auf längere Sicht könnte die elastokapillare Selbstorganisation von Nanofilamenten den Aufbau extrazellulärer Matrizes für die Gewebezüchtung ermöglichen (Abbildung 11d)..$^{[13,92]}$

Energie: Die Verwendung der kapillaren Selbstorganisation an dicht gepackten Nanofilamenten ist aufgrund des Bedarfs einer hochspezifischen Oberfläche auch für Superkondensatorelektroden von Nutzen. ${ }^{[3]}$ Futuba et al. bauten z.B. Doppelschichtkondensator(EDLC)-Zellen aus dicht ausgerichteten CNT-Wäldern, die eine spezifische Kapazität von $80 \mathrm{Fg}^{-1}$ für eine Drei-Elektroden-Zelle lieferten. ${ }^{[3]}$ Erst kürzlich wurden in der gleichen Gruppe DünnschichtSWCNT-Kondensatoren erzeugt, in denen mechanisches
Scheren mit einer kapillaren Selbstorganisation kombiniert wurde, was zu einer Kapazität von $160 \mathrm{Fg}^{-1}$ führte (Abbildung $11 \mathrm{e}) .^{[2]}$

Pint et al. verwendeten die kapillare Selbstorganisation zur Herstellung von Antireflexoberflächen für photoelektrochemische Bauelemente. ${ }^{[6]}$ Sie stellten CNF-Arrays mit einer Top-Gathering-Anordnung mittels Kondensation her und beschichteten diese dann mittels ALD mit $\mathrm{TiO}_{2}$-Schichten. Dabei wurde für die kapillar texturierten photoelektrochemischen $\mathrm{CNT} / \mathrm{TiO}_{2}$-Bauelemente im Vergleich zu einem planaren Äquivalent eine bis zu 3-fach bessere KurzschlussStromdichte nachgewiesen.

Außerdem können die hierarchischen und robusten Oberflächentexturen, die durch eine kapillare Selbstorganisation hergestellt werden, zur Entwicklung eines Wärmetransfers mit Phasenumwandlung von Nutzen sein. Kapillar selbstorganisierte Nanodraht-Arrays aus $\mathrm{Si}$ und $\mathrm{Cu}$ zeigten höhere Wärmeübergangskoeffizienten und eine um mehr als $100 \%$ erhöhte kritische Wärmestromdichte verglichen mit unstrukturierten Substraten. ${ }^{[38]}$ Dabei sollten die schaumähnlichen Mikrohohlräume mit einer Breite von 1-6 $\mu$ m nach Ansicht der Autoren den Keim für die Bildung von Blasen während des Siedens bilden (Abbildung $7 \mathrm{a}, \mathrm{b}$ ). Experimente zu einem besseren Sieden bei freier Konvektion wurden auch mit CNT-Wäldern durchgeführt, obwohl dabei unklar ist, ob die Nanofilamente vor dem Sieden einem kapillaren Selbstorganisationsschritt unterzogen wurden. ${ }^{[93]}$

Darüber hinaus existieren weitere Anwendungsvorschläge, wie die Verwendung der elastokapillaren Aggregation zur Modulierung der Farbe der Bragg-Beugung in Hydrogel-Mikrosäulen-Anordnungen (Abbildung 12d) ${ }^{[1,42]}$ oder die Nutzung von selbstorganisierten CNT-Schäumen zur stoßabsorbierenden Strukturverstärkung, ${ }^{[23]}$ für elastische Membranen, ${ }^{[23]}$ Filtrationsbauelemente, ${ }^{[23,94]}$ Bürstenmotoren, ${ }^{[69]}$ Temperatursensoren ${ }^{[34]}$ und komplexe Masterformen. ${ }^{[20,35]}$

\section{Zusammenfassung und Ausblick}

Die elastokapillare Aggregation von mikro- und nanoskaligen Filamenten tritt sowohl bei natürlichen als auch synthetischen Materialien auf, und das Verhalten von Filamenten in Flüssigkeiten ist sowohl für deren Verarbeitung als auch deren Nutzung in einer Vielzahl von Anwendungen wesentlich. Während die elastokapillare Aggregation anfangs eher als Ärgernis bei der Gestaltung von kleinen, flexiblen Texturen auf Siliciumwafern betrachtet wurde, wird sie heute als Mittel zur Herstellung komplexer, hierarchischer Oberflächenstrukturen gesehen. Insbesondere ist dieser Prozess inhärent kostengünstig und skalierbar und nutzt den Vorteil von wohlbekannten planaren Strukturierungsmethoden, um neue Geometrien zu schaffen, die durch andere Methoden nicht zugänglich sind. Jüngste Arbeiten zeigen, dass die hierarchischen Anordnungen von Nanofilamenten insbesondere hinsichtlich der Schaffung von Materialien mit großer Oberfläche für Energieanwendungen sowie zur Herstellung von Oberflächentexturen mit kontrollierbaren Benetzungs-, Adhäsions-, optischen oder elektrischen Eigenschaften großes Potenzial bieten. Weitere Entwicklungen bei der 
Herstellung von Nanofilamenten werden die Vielseitigkeit der elastokapillaren Selbstorganisation über die heute am häufigsten verwendeten Materialien hinaus erweitern, und die Herstellung großflächiger Nanoelemente wird auch Eingang in die Industrie finden.

M.D.V. wurde durch den Fund for Scientific Research Flanders FWO und IAP- $\mu$ MAST Belspo (Belgien) unterstützt. A.J.H. wurde durch das Young Investigator Program des Air Force Office of Scientific Research gefördert (FA9550-11-10089, Programmleiter Dr. Byung-Lip „Les“ Lee). Wir danken Sameh Tawfick für hilfreiche Diskussionen und Beiträge zu Abbildung 9.

Eingegangen am 25. Juli 2012

Online veröffentlicht am 21. Januar 2013

Übersetzt von Dr. Christiane Feldmann-Leben, Ettlingen

[1] D. Chandra, S. Yang, Acc. Chem. Res. 2010, 43, 1080-1091.

[2] A. Izadi-Najafabadi, S. Yasuda, K. Kobashi, T. Yamada, D. N. Futaba, H. Hatori, M. Yumura, S. Iijima, K. Hata, Adv. Mater. 2010, 22, E235-E241.

[3] D. N. Futaba, K. Hata, T. Yamada, T. Hiraoka, Y. Hayamizu, Y. Kakudate, O. Tanaike, H. Hatori, M. Yumura, S. Iijima, Nat. Mater. 2006, 5, 987-994.

[4] H. J. Gao, X. Wang, H. M. Yao, S. Gorb, E. Arzt, Mech. Mater 2005, 37, 275-285.

[5] L. Qu, L. Dai, M. Stone, Z. Xia, Z. L. Wang, Science 2008, 322 , $238-242$.

[6] C. L. Pint, K. Takei, R. Kapadia, M. Zheng, A. C. Ford, J. Zhang, A. Jamshidi, R. Bardhan, J. J. Urban, M. Wu, J. W. Ager, M. M. Oye, A. Javey, Adv. Energy Mater. 2011, 1, 1040-1045.

[7] G. K. Mor, K. Shankar, M. Paulose, O. K. Varghese, C. A. Grimes, Nano Lett. 2006, 6, 215-218.

[8] K. K. S. Lau， J. Bico，K. B. K. Teo, M. Chhowalla， G. A. J. Amaratunga, W. I. Milne, G. H. McKinley, K. K. Gleason, Nano Lett. 2003, 3, $1701-1705$.

[9] N. Mingo, D. A. Broido, Phys. Rev. Lett. 2005, 95, 096105.

[10] N. Kaji, Y. Tezuka, Y. Takamura, M. Ueda, T. Nishimoto, H. Nakanishi, Y. Horiike, Y. Baba, Anal. Chem. 2004, 76, 15-22.

[11] F. Fachin, G. D. Chen, M. Toner, B. L. Wardle, J. Microelectromech. Syst. 2011, 20, 1428-1438.

[12] T. D. B. Nguyen-Vu, H. Chen, A. M. Cassell, R. Andrews, M. Meyyappan, J. Li, Small 2006, 2, 89-94.

[13] M. A. Correa-Duarte, N. Wagner, J. Rojas-Chapana, C. Morsczeck, M. Thie, M. Giersig, Nano Lett. 2004, 4, 2233-2236.

[14] S. Tawfick, M. De Volder, D. Copic, S. J. Park, C. R. Oliver, E. S. Polsen, M. J. Roberts, A. J. Hart, Adv. Mater. 2012, 24, 1628 1674.

[15] T. Tanaka, M. Morigami, N. Atoda, Jpn. J. Appl. Phys. Part 1 1993, 32, 6059-6064.

[16] A. Grinthal, S. H. Kang, A. K. Epstein, M. Aizenberg, M. Khan, J. Aizenberg, Nano Today 2012, 7, 35-52.

[17] Z. C. Liu, L. J. Ci, S. Kar, P. M. Ajayan, J. Q. Lu, IEEE Trans. Nanotechnol. 2009, 8, 196-203.

[18] E. Verploegen, A. J. Hart, M. De Volder, S. Tawfick, K. K. Chia, R. E. Cohen, J. Appl. Phys. 2011, 109, 094316.

[19] M. F. L. De Volder, S. J. Park, S. H. Tawfick, D. O. Vidaud, A. J. Hart, J. Micromech. Microeng. 2011, 21, 045033.

[20] D. Copic, S. J. Park, S. Tawfick, M. F. L. De Volder, A. J. Hart, Lab Chip 2011, 11, 1831-1837.

[21] C. Journet, S. Moulinet, C. Ybert, S. T. Purcell, L. Bocquet, Europhys. Lett. 2005, 71, 104-109.
[22] D. Copic, S. J. Park, S. Tawfick, M. De Volder, A. J. Hart, J. Visualized Exp. 2012, 65, e3980.

[23] N. Chakrapani, B. Q. Wei, A. Carrillo, P. M. Ajayan, R. S. Kane, Proc. Natl. Acad. Sci. USA 2004, 101, 4009-4012.

[24] M. De Volder, S. H. Tawfick, S. J. Park, D. Copic, Z. Z. Zhao, W. Lu, A. J. Hart, $A d v$. Mater. 2010, 22, 4384-4389.

[25] H. Liu, S. H. Li, J. Zhai, H. J. Li, Q. S. Zheng, L. Jiang, D. B. Zhu, Angew. Chem. 2004, 116, 1166-1169; Angew. Chem. Int. Ed. 2004, 43, 1146-1149.

[26] S. Tawfick, M. De Volder, A. J. Hart, Langmuir 2011, 27, 63896394.

[27] Y. Hayamizu, T. Yamada, K. Mizuno, R. C. Davis, D. N. Futaba, M. Yumura, K. Hata, Nat. Nanotechnol. 2008, 3, 289-294.

[28] S. Tawfick, A. J. Hart, M. De Volder, Nanoscale 2012, 4, 3852 3856.

[29] Y. Hayamizu, R. C. Davis, T. Yamada, D. N. Futaba, S. Yasuda, M. Yumura, K. Hata, Phys. Rev. Lett. 2009, 102, 175505.

[30] E. R. Meshot, K. D. Patel, S. Tawfick, K. A. Juggernauth, M. Bedewy, E. A. Verploegen, M. F. L. De Volder, A. J. Hart, $A d v$. Funct. Mater. 2012, 22, 577-584.

[31] M. F. L. De Volder, D. O. Vidaud, E. R. Meshot, S. Tawfick, A. J. Hart, Microelectron. Eng. 2010, 87, 1233-1238.

[32] Siehe Lit. [24].

[33] M. F. L. De Volder, S. Tawfick, S. J. Park, A. J. Hart, ACS Nano 2011, $5,7310-7317$.

[34] M. De Volder, D. Reynaerts, C. Van Hoof, S. Tawfick, A. J. Hart, IEEE Sensors 2010, 2369-2372.

[35] Lit. [22].

[36] H. Cai, Y. Xu, P. G. He, Y. Z. Fang, Electroanalysis 2003, 15, $1864-1870$.

[37] Y. P. Zhao, J. G. Fan, Appl. Phys. Lett. 2006, 88, 103123.

[38] R. Chen, M.-C. Lu, V. Srinivasan, Z. Wang, H. H. Cho, A. Majumdar, Nano Lett. 2009, 9, 548-553.

[39] H. Namatsu, K. Kurihara, M. Nagase, K. Iwadate, K. Murase, Appl. Phys. Lett. 1995, 66, 2655-2657.

[40] J. J. Hill, K. Haller, B. Gelfand, K. J. Ziegler, ACS Appl. Mater. Interfaces 2010, 2, 1992-1998.

[41] A. Dev, S. Chaudhuri, Nanotechnology 2007, 18, 175607.

[42] D. Chandra, S. Yang, A. A. Soshinsky, R. J. Gambogi, ACS Appl. Mater. Interfaces 2009, 1, 1698-1704.

[43] H. Duan, K. K. Berggren, Nano Lett. 2010, 10, 3710-3716.

[44] H. Duan, J. K. W. Yang, K. K. Berggren, Small 2011, 7, 2661 2668.

[45] A. Ishikawa, M. Sakata, A. Kawai, J. Photopolym. Sci. Technol. 2004, 17, 457-460.

[46] B. Chen, S. Seidel, H. Hori, M. Gupta, ACS Appl. Mater. Interfaces 2011, 3, $4201-4205$.

[47] S. Choi, M. Yan, I. Adesida, Appl. Phys. Lett. 2008, 93, 163113.

[48] D. E. Noga, R. A. Lawson, C.-T. Lee, L. M. Tolbert, C. L. Henderson in Conference on Advances in Resist Materials and Processing Technology XXVI, San Jose, CA, 2009.

[49] J. Bico, B. Roman, L. Moulin, A. Boudaoud, Nature 2004, 432, $690-690$

[50] M. Mastrangeli, S. Abbasi, C. Varel, C. Van Hoof, J. P. Celis, K. F. Boehringer, J. Micromech. Microeng. 2009, 19, 045015.

[51] B. Roman, J. Bico, J. Phys. Condens. Matter 2010, 22, 493101.

[52] M. Grzelczak, J. Vermant, E. M. Furst, L. M. Liz-Marzan, ACS Nano 2010, 4, 3591-3605.

[53] G. M. Whitesides, B. Grzybowski, Science 2002, 295, 2418-2421.

[54] N. Vogel, C. K. Weiss, K. Landfester, Soft Matter 2012, 8, $4044-$ 4061.

[55] B. Pokroy, S. H. Kang, L. Mahadevan, J. Aizenberg, Science 2009, 323, 237-240.

[56] S. H. Kang, B. Pokroy, L. Mahadevan, J. Aizenberg, Acs Nano 2010, 4, 6323-6331.

[57] M. Matsunaga, M. Aizenberg, J. Aizenberg, J. Am. Chem. Soc. 2011, 133, 5545-5553. 


\section{$\underset{\text { Angewandte }}{\text { Aufätze }}$}

[58] P. Kim, W. E. Adorno-Martinez, M. Khan, J. Aizenberg, Nat. Protoc. 2012, 7, 311-327.

[59] M. F. L. De Volder, R. Vansweevelt, P. Wagner, D. Reynaerts, C Van Hoof, A. J. Hart, ACS Nano 2011, 5, 6593-6600.

[60] C. L. Cheung, R. J. Nikolic, C. E. Reinhardt, T. F. Wang, Nanotechnology 2006, 17, 1339-1343.

[61] A. J. Hart, A. H. Slocum, J. Phys. Chem. B 2006, 110, $8250-$ 8257.

[62] K. Hata, D. N. Futaba, K. Mizuno, T. Namai, M. Yumura, S. Iijima, Science 2004, 306, 1362-1364.

[63] Siehe Lit. [31]

[64] Siehe Lit. [25].

[65] X. D. Lim, H. W. G. Foo, G. H. Chia, C. H. Sow, ACS Nano 2010, 4, $1067-1075$

[66] J. Liang, R. G. D. Jeyasingh, H.-Y. Chen, H. S. P. Wong, IEEE Trans. Electron Devices 2012, 59, 1155-1163.

[67] M. De Volder, J. Decoster, D. Reynaerts, C. Van Hoof, S.-G. Kim, Small 2012, 8, 2006.

[68] Z. Liu, N. Bajwa, L. Ci, S. H. Lee, S. Kar, P. M. Ajayan, et al., 10th International Interconnect Technology Conference 2007, S. $201-203$.

[69] D. N. Futaba, K. Miyake, K. Murata, Y. Hayamizu, T. Yamada, S. Sasaki, M. Yumura, K. Hata, Nano Lett. 2009, 9, 3302-3307.

[70] Siehe Lit. [30]

[71] A. Kim, F. S. Ou, D. A. A. Ohlberg, M. Hu, R. S. Williams, Z. Li, J. Am. Chem. Soc. 2011, 133, 8234-8239.

[72] G. Liu, J. Zhou, Y. Xiong, X. Zhang, Y. Tian, Nanotechnology 2011, 22, 305305.

[73] M. De Volder, S. Tawfick, S. J. Park, D. Copic, A. J. Hart, TRANSDUCERS 2011-2011 16th International Solid-State Sensors, Actuators and Microsystems Conference, 01, 2011.

[74] S. Esconjauregui, M. Fouquet, B. C. Bayer, C. Ducati, R. Smajda, S. Hofmann, J. Robertson, ACS Nano 2010, 4, 7431-7436.

[75] D. Wu, Q.-D. Chen, B.-B. Xu, J. Jiao, Y. Xu, H. Xia, H. B. Sun, Appl. Phys. Lett. 2009, 95, 091902.

[76] F. Járai-Szabó, E.-A. Horvat, R. Vajtai, Z. Neda, Chem. Phys. Lett. 2011, 511, 378-383.
[77] Siehe Lit. [22].

[78] Z. Z. Zhao, S. H. Tawfick, S. J. Park, M. De Volder, A. J. Hart, W. Lu, Phys. Rev. E 2010, 82, 041605.

[79] J. Qu, Z. Zhao, X. Wang, J. Qiu, J. Mater. Chem. 2011, 21, $5967-$ 5971.

[80] S. H. Kang, N. Wu, A. Grinthal, J. Aizenberg, Phys. Rev. Lett. 2011, 107, 177802.

[81] N. Chiodarelli, Y. Li, D. J. Cott, S. Mertens, N. Peys, M. Heyns, S. D. Gendt, G. Groeseneken, P. M. Vereecken, Microelectron. Eng. 2011, 88, 837-843.

[82] T. Wang, S. Chen, D. Jiang, Y. Fu, K. Jeppson, L. Ye, J. Liu, IEEE Electron Device Lett. 2012, 33, 420-422.

[83] M. De Volder, S. H. Tawfick, D. Copic, A. J. Hart, Soft Matter 2011, 7, $9844-9847$.

[84] M. De Volder, S. Tawfick, D. Copic, A. J. Hart, TRANSDUCERS 2011-2011 16th International Solid-State Sensors, Actuators and Microsystems Conference, 012011.

[85] H. Segawa, S. Yamaguchi, Y. Yamazaki, T. Yano, S. Shibata, H. Misawa, Appl. Phys. A 2006, 83, 447-451.

[86] H. Segawa, Y. Yamazaki, T. Yano, S. Shibata, Mater. Sci. Eng. B 2008, 148, 43-47.

[87] H. Segawa, Y. Yamazaki, S. Tachiki, T. Yano, S. Shibata, J. Colloid Interface Sci. 2008, 323, 187-190.

[88] H. Segawa, S. Tachiki, T. Yano, S. Shibata, Mater. Sci. Eng. B 2009, 161, 85-90.

[89] H. Segawa, D. Morishima, T. Yano, S. Shibata, Mater. Sci. Eng. B 2010, 173, $162-167$.

[90] M. Hu, F. S. Ou, W. Wu, I. Naumov, X. Li, A. M. Bratkovsky, R. S. Williams, Z. Y. Li, J. Am. Chem. Soc. 2010, 132, 1282012822 .

[91] S. Barcelo, A. Kim, W. Wu, Z. Li, ACS Nano 2012, 6, 6446-6452.

[92] T. Dvir, B. P. Timko, D. S. Kohane, R. Langer, Nat. Nanotechnol. 2011, $6,13-22$.

[93] H. S. Ahn, N. Sinha, M. Zhang, D. Banerjee, S. Fang, R. H. Baughman, J. Heat Transfer 2006, 128, 1335-1342.

[94] M. Yu, H. H. Funke, J. L. Falconer, R. D. Noble, Nano Lett. 2009, 9, 225-229. 